\title{
Myocardial infarction triggers cardioprotective antigen- specific T helper cell responses
}

\author{
Max Rieckmann, ${ }^{1}$ Murilo Delgobo, ${ }^{2,3}$ Chiara Gaal, ${ }^{2,3}$ Lotte Büchner, ${ }^{2,3}$ Philipp Steinau, ${ }^{1}$ Dan Reshef, ${ }^{4}$ Cristina Gil-Cruz, ${ }^{5}$ \\ Ellis N. ter Horst, ${ }^{6,7,8,9}$ Malte Kircher, ${ }^{10}$ Theresa Reiter, ${ }^{2,3}$ Katrin G. Heinze, ${ }^{11}$ Hans W.M. Niessen,, Paul A.J. Krijnen, ${ }^{7,8}$ \\ Anja M. van der Laan, ${ }^{6}$ Jan J. Piek, ${ }^{6,8}$ Charlotte Koch, ${ }^{1}$ Hans-Jürgen Wester, ${ }^{12}$ Constantin Lapa, ${ }^{10}$ Wolfgang R. Bauer, ${ }^{2,3}$ \\ Burkhard Ludewig, ${ }^{5}$ Nir Friedman, ${ }^{4}$ Stefan Frantz, ${ }^{1,2,3}$ Ulrich Hofmann,, 1,2,3 and Gustavo Campos Ramos ${ }^{1,2,3}$ \\ 'Department of Internal Medicine III, University Clinic Halle, Halle, Germany. ${ }^{2}$ Department of Internal Medicine I, and ${ }^{3}$ Comprehensive Heart Failure Center, University Hospital Würzburg, Würzburg, \\ Germany. ${ }^{4}$ Department of Immunology, Weizmann Institute of Science, Rehovot, Israel. Institute of Immunobiology, Kantonsspital St. Gallen, St. Gallen, Switzerland. ${ }^{6} \mathrm{Heart}$ Center, Amsterdam UMC, \\ location AMC, Amsterdam, Netherlands. 'Department of Pathology and Cardiac Surgery, Amsterdam UMC, location VUmc, Amsterdam, Netherlands. ${ }^{8}$ Amsterdam Cardiovascular Sciences, Amsterdam UMC, \\ Amsterdam, Netherlands. ${ }^{9}$ Netherlands Heart Institute, Utrecht, Netherlands. ${ }^{10}$ Department of Nuclear Medicine, University Hospital Würzburg, Würzburg, Germany. ${ }^{11}$ Rudolf Virchow Center for Experimental \\ Biomedicine, University of Würzburg, Würzburg, Germany. ${ }^{12}$ Pharmaceutical Radiochemistry, Technical University Munich, Munich, Germany.
}

T cell autoreactivity is a hallmark of autoimmune diseases but can also benefit self-maintenance and foster tissue repair. Here, we investigated whether heart-specific T cells exert salutary or detrimental effects in the context of myocardial infarction (MI), the leading cause of death worldwide. After screening more than 150 class II-restricted epitopes, we found that myosin heavy chain $\alpha$ (MYHCA) was a dominant cardiac antigen triggering post-MI CD4+ $\mathrm{T}$ cell activation in Balb/c mice. Transferred MYHCA $_{614-629}$-specific CD4+ $T$ cells (TCR-M cells) selectively accumulated in the myocardium and mediastinal lymph nodes (med-LNs) of infarcted mice, acquired a Treg phenotype with a distinct prohealing gene expression profile, and mediated cardioprotection. Myocardial Tregs were also detected in autopsy samples from patients who had had a MI. Noninvasive PET/CT imaging using a CXCR4 radioligand revealed enlarged med-LNs with increased cellularity in patients with MI. Notably, the med-LN alterations observed in MI patients correlated with the infarct size and cardiac function. Taken together, the results obtained in our study provide evidence that MI context induces prohealing $\mathrm{T}$ cell autoimmunity in mice and confirm the existence of an analogous heart/med-LN/T cell axis in patients with MI.

\section{Introduction}

Cardiology and immunology are disciplines born apart, with different goals and tenets. Nonetheless, a fertile association between these areas of knowledge has recently flourished, and leukocytes have now been implicated in the control of cardiac metabolism (1), rhythm $(2,3)$, aging (4), and repair (5-8). The contribution of immunological phenomena to cardiac repair is of particular clinical relevance, because myocardial infarction (MI) is the leading cause of death worldwide (9). In addition to causing a life-threatening hemodynamic imbalance, the myocardial injury triggered by ischemia imposes a healing challenge for the heart, which is a terminally differentiated organ. Thus, many patients who survive an acute MI episode subsequently develop chronic heart failure (HF) due to maladaptive myocardial repair (10). Hence, there is an unmet need to better understand and

\section{Related Commentary: p. 4587}

Authorship note: SF, UH, and GCR share senior authorship. Conflict of interest: HJW is the founder and a shareholder of Scintomics, which provided the CXCR4 radiotracer.

Copyright: (5) 2019, American Society for Clinical Investigation.

Submitted: July 30, 2018; Accepted: August 8, 2019; Published: October 14, 2019. Reference information: J Clin Invest. 2019;129(11):4922-4936.

https://doi.org/10.1172/JCl123859. eventually modulate the immune-inflammatory mechanisms underlying the myocardial healing process.

$\mathrm{T}$ lymphocytes are major players in the mediation of tissue repair, including that following cardiac injury (11-14). The participation of $\mathrm{T}$ cells in myocardial inflammation and repair has been observed in many organisms, ranging from fishes (14) to mammals (15-19), indicating that it is an evolutionarily conserved phenomenon. Previous studies from our group and others indicated that $\mathrm{CD}^{+} \mathrm{T}$ cells become primed and proliferate in the mediastinal lymph nodes (med-LNs) (heart-draining LNs) of infarcted mice and then infiltrate the myocardium $(16,18,20,21)$. Independent groups have reported that the Tregs (defined as FOXP3 ${ }^{+}$) activated after MI exert salutary effects by accelerating the resolution of inflammation and promoting appropriate extracellular matrix deposition in the myocardial scar $(14,18,20,22,23)$. Conventional T helper (Tconv) cells (defined as FOXP3-) have also been shown to positively influence the molecular milieu in the infarcted myocardium via a mechanism that depends on the extracellular purinergic metabolism (24).

$\mathrm{T}$ cell reactivity can benefit myocardial healing in mice by promoting reparative fibrosis in a postmitotic organ. However, sustained $\mathrm{T}$ cell responses in the heart can also fuel adverse remodeling and contribute to the progression of ischemic $\mathrm{HF}$ at later chronic stages (19). Furthermore, chronic T cell activation in the myocardium has also been shown to be detrimental in experimen- 
tal models of pressure overload-induced HF and during aging (4, 25-27). Taken together, these findings indicate that the crosstalk between $T$ cells and the heart can result in a broad spectrum of possible outcomes in mice, depending on the context of activation and the timing of these responses. Furthermore, whether post-MI T cell responses are driven by cardiac antigens or by the recognition of danger-associated molecular patterns in the context of a particular cytokine milieu has not yet been established. Most important, the translational relevance of this heart/T cell axis has not yet been fully confirmed in a meaningful clinical setting, mainly due to the challenges of obtaining samples from relevant sites other than peripheral blood, such as the myocardium and draining LNs (28).

In this study, we sought to elucidate the principles governing $\mathrm{T}$ cell activation, differentiation, and functionality in the context of experimental MI (EMI) and to monitor the distribution of T cells in the heart and med-LNs of patients with MI. Through a combination of unbiased screening with hypothesis-driven approaches, we identified an antigen-specific mode of $T$ cell activation and revealed that the myocardium imposes an activation context that promotes the development of heart-specific T helper (Th) cells poised to exhibit a cardioprotective phenotype during the healing stage. Moreover, we combined histological analyses of myocardial autopsy samples with thoracic PET/CT imaging using the novel radiotracer $\left[{ }^{68} \mathrm{Ga}\right]$ pentixafor, a ligand for CXCR4, and our results provide strong clinical evidence that a heart/ $\mathrm{T}$ cell axis is also active in MI patients.

\section{Results}

Myosin heavy chain $\alpha$ is a dominant cardiac antigen that triggers Th cell activation after EMI. The proteins released by cardiomyocyte death during an ischemic event can be a source of antigens that stimulate adaptive immune cells. Thus, we sought to verify whether post-MI CD $4^{+} \mathrm{T}$ cell responses are heart specific and to ultimately identify relevant cardiac epitopes. We tested the reactivity profile of $\mathrm{CD}^{+} \mathrm{T}$ cells purified from infarcted mice against a library of 153 peptides covering the 8 most relevant heart-enriched proteins encoded by mRNAs with more than 5 levels in the heart compared with those in all other tissues, as detailed in the Human Protein Atlas (29). The cardiac proteins selected include sarcomere elements ( $\alpha$ actin, cardiac muscle 1 [ACTC1], myosin-binding protein C3 [MYBPC3], myosin heavy chain $\alpha$ [MYHCA], myosin light chain 2 [MYL2], troponin I3, cardiac muscle [TNNI3], and troponin T2, cardiac muscle [TNNT2]), a cardiac receptor (adrenergic receptor $\beta 1$ [ARB1]), and a mitochondrial component (heat shock protein family B3 [HSPB3]). The specific peptides (15 mers) (Supplemental Table 1; supplemental material available online with this article; https://doi.org/10.1172/JCI123859DS1) were selected on the basis of in silico simulation of their MHC-II binding properties (30).

As shown in Figure 1, A-E, splenocytes purified from infarcted (but not from sham-operated) mice showed an IL-2 response to MYHCA-derived epitopes, which is considered a readout for antigen-specific stimulation. No IL-2 response was observed when the $\mathrm{T}$ cells were challenged with all other tested peptides, revealing that MYHCA is a dominant cardiac antigen in the MI context. This heart-specific myosin isoform has already been implicated in the pathogenesis of experimental autoimmune myocarditis, and previous studies identified a specific MHC-II-restricted epitope $\left(\right.$ MYHCA $\left._{614-628}\right)$ that is relevant in that disease model $(31,32)$. As shown in Figure 1, A, C, and E, this single MYHCA $_{614-628}$ peptide could recapitulate similar $\mathrm{T}$ cell responses in infarcted mice. The results revealed no response to a control peptide representing an irrelevant antigen $\left(\mathrm{OVA}_{323-339}\right)$, and we observed no IFN- $\gamma$ response to any of the peptides tested, including MYHCA (Figure 1A).

Cardiac myosin-specific Th cells selectively accumulate in the infarcted heart and acquire a regulatory phenotype. After identifying MYHCA $_{614-628}$ as a crucial cardiac antigen that triggers the activation of Th cells in the MI context, we sought to monitor the in vivo distribution and activation profile of MYHCA-specific $\mathrm{T}$ cells in infarcted mice. To that end, we adoptively transferred $5 \times 10^{6}$ Thy1.1 MYHCA-specific cells into Thy1.2 syngeneic WT recipient mice prior to the induction of EMI and monitored the distribution and activation profile of these cells by flow cytometry (Figure 2 and Supplemental Figure 1). We obtained these cells from a mouse strain exclusively bearing transgenic $\mathrm{T}$ cell receptors (TCRs) specific for the immunogenic MYHCA peptide $\left(\right.$ MYHCA $_{614-629}$ ) presented in the MHC-II context (hereafter referred to as TCR-M cells) (33). Monoclonal TCR-M cells were defined as $\mathrm{CD} 4^{+} \mathrm{TCR} \beta^{+} \mathrm{Thy} 1.1^{+} \mathrm{TCV} \alpha 2^{+}$singlets. Polyclonal endogenous Th cells (ENDO cells) were defined as CD $4^{+}$TCR $\beta^{+}$Thy $1.1^{-}$ singlets. As shown in Figure 2B, TCR-M cells selectively accumulated in the heart and med-LNs of infarcted mice at the peak of the wound-healing phase (day 7). The TCR-M cells vanished during a later chronic phase, indicating that post-MI autoreactivity to cardiac myosin is a self-limiting phenomenon (Figure 2C). Light-sheet fluorescence microscopy (LSFM) of whole unsliced hearts confirmed that TCR-M cells accumulated within the infarct zone but not in the healthy remote myocardium (Figure 2, D and E, and Supplemental Video 1). The results revealed no antigenspecific T cell accumulation on day 49 after MI (Figure 2F).

MI also had a major impact on the differentiation of TCR-M cells. When transferred into the MI context, TCR-M cells exhibited increased levels of CD44 surface expression at all sites compared with baseline (i.e., before transfer) expression levels (Figure 2, G-I). The frequency of $\mathrm{CD} 44^{+}$differentiated cells was also higher among MI TCR-M cells than in the endogenous $\mathrm{CD}^{+} \mathrm{T}$ cell compartment (ENDO cells) at all analyzed sites $(P<0.05)$. We observed no CD44 upregulation in the TCR-M cells found in the subiliac LNs (si-LNs) of sham-operated mice (Figure 2G). However, the TCR-M cells isolated from the med-LNs of both infarcted and sham-operated mice displayed markers of cell activation (Figure $2 \mathrm{H}$ ). These findings suggest that MYHCA is constitutively presented to $\mathrm{CD}^{+} \mathrm{T}$ cells in the heart-draining LNs of healthy mice, even in the absence of cardiac damage, as previously reported $(21,34)$. TCR-M cells were virtually absent from sham-operated hearts, but they exhibited a differentiated phenotype in infarcted hearts (Figure 2I). We observed a similar pattern for FOXP3 expression. Notably, 55.2\% of the TCR-M cells in the infarcted hearts were $\mathrm{FOXP}^{+}$, in sharp contrast to the baseline frequency (8.8\%).

To better understand the mechanisms underlying the accumulation of $\mathrm{FOXP3}^{+}$TCR-M cells in the heart, we transferred Treg $\left(\mathrm{CD}^{2} 5^{+}, \mathrm{FOXP3}^{+}\right)$and Tconv (CD25-, FOXP3-) TCR-M cells labeled with distinct, subset-specific fluorescent intracellular tracers (Figure 3A). Flow cytometric analyses performed on day 7 after MI confirmed that both $\mathrm{T}$ cell subsets strongly proliferated in the heart and the med-LNs, but in not the si-LNs, of infarcted mice 

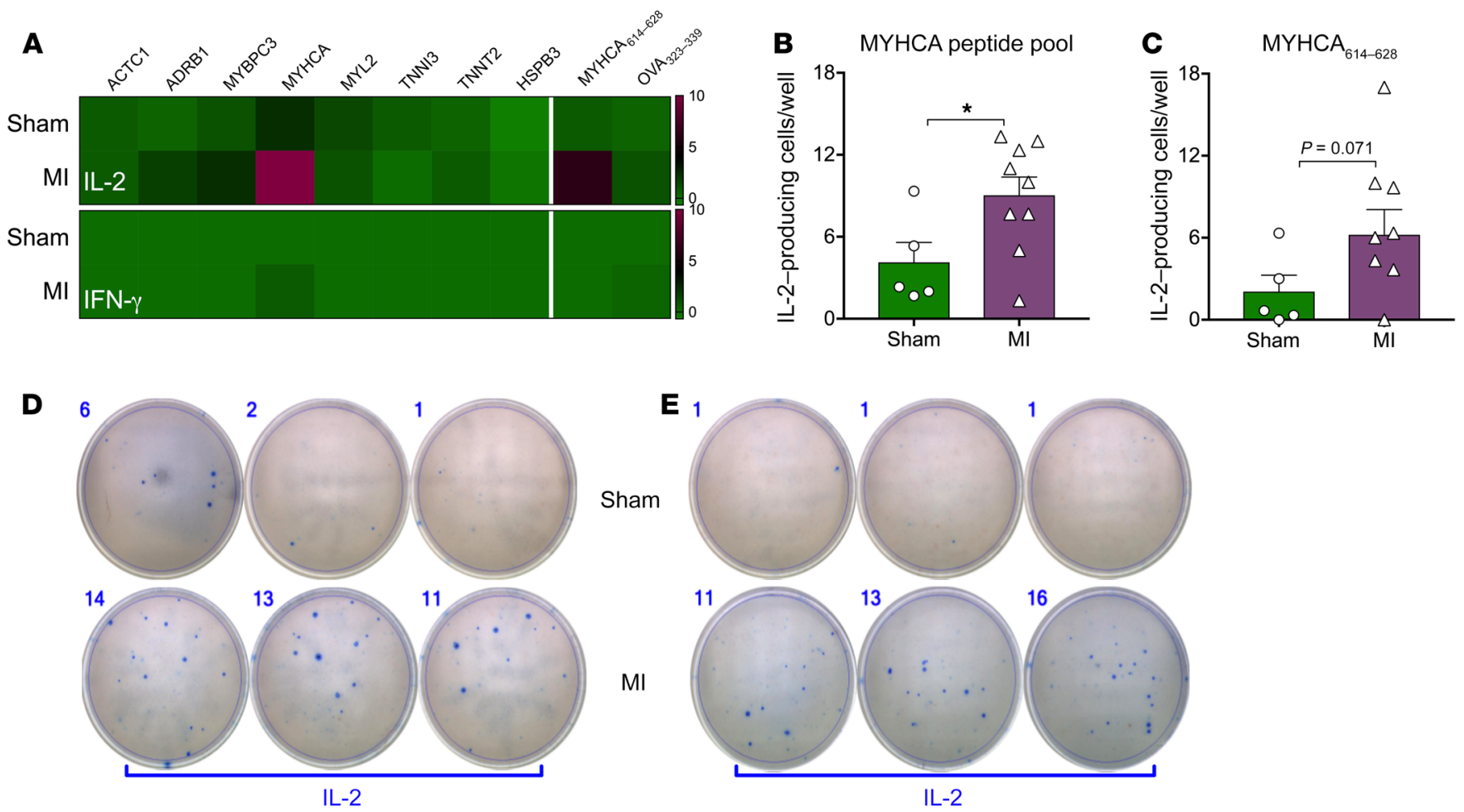

Figure 1. Cardiac epitope mapping. (A) Heatmaps depicting the number of specific T cell responses to defined antigens of interest. Splenocytes purified from MI or sham-operated mice (day 7) were cultured in the presence of peptide pools (15 mers, MHC-II-restricted) covering the most important heart-enriched proteins, and the production of IL-2 and IFN- $\gamma$ was monitored by ELISPOT as a readout for antigen-specific stimulation. The single peptides MYHCA $_{614-628}$ (included in the MYHCA pool) and OVA $_{323-339}$ (irrelevant antigen, not expressed in the heart) were also tested. (B and $\mathbf{C}$ ) Quantification of IL-2-producing cells per well in response to (B) the MYHCA peptide pool and (C) MYHCA 614-628. $^{\circ}$ ( $\mathbf{D}$ and $\left.\mathbf{E}\right)$ Representative ELISPOT images of these antigens. The number of responder cells found in each well is shown directly next to the representative images. The bar graphs display the group mean values, the SEM, and the distribution of each individual value. Statistical analysis was determined with a 2-tailed, unpaired $t$ test $(\mathbf{B}$ and $\mathbf{C})$. ${ }^{*} P<0.05$. The data were acquired from 3 independent experiments.

(Figure 3, B-D). These results confirm that post-MI T cell responses are largely driven by MYHCA-derived antigens. Remarkably, more than $50 \%$ of the TCR-M cells labeled as Tconv prior to cell transfer acquired FOXP3 ${ }^{+}$expression in the myocardium, but not in the LNs, indicating that in situ Treg conversion was the major factor driving Treg accumulation in the myocardial tissue (Figure 3B). Myosin-specific T cells also underwent in situ Treg conversion in the absence of MI (Figure 3B), but the numbers of TCR-M cells found in the infarcted hearts were typically higher than those in sham-operated hearts (Figure 2, B and L). Taken together, these results indicate that MI promotes the activation and recruitment of heart-specific Th cells and that the myocardial context induces myosin-specific $\mathrm{T}$ cells to acquire a Treg phenotype.

Antigen-specific Th cells activated in the MI context acquire a nonclassical gene expression signature enriched with prohealing factors. To better characterize how the MI context shapes the differentiation of antigen-specific $\mathrm{CD} 4^{+} \mathrm{T}$ cells, we subsequently sorted TCR-M and ENDO cells from the med-LNs of infarcted and sham-operated mice (day 7) for downstream gene expression profiling (Figure 4 and Supplemental Figure 2).

The TCR-M cells were purified by FACS and defined as $\mathrm{CD} 4{ }^{+} \mathrm{TCR} \beta^{+} \mathrm{Thy} 1.1^{+} \mathrm{TCV} \alpha 2^{+}$singlets, whereas the ENDO cells were defined as $\mathrm{CD} 4^{+} \mathrm{TCR} \beta^{+}$Thy $1.1^{-}$singlets. As shown in Figure 4, A and B, the TCR-M and ENDO cell compartments displayed contrasting gene expression patterns in response to MI. After MI, the ENDO compartment, primarily composed of polyclonal cells, showed 236 downregulated genes but only 43 upregulated genes ( $>2$-fold difference versus the sham counterpart, $P<0.05$ ). These findings reveal that, despite patent inflammation, $\mathrm{MI}$ imposes a largely inhibitory context on most polyclonal T cells. In sharp contrast, the TCR-M cells had 153 upregulated and only 38 downregulated transcripts in response to MI, confirming that MI promotes the stimulation of heart-specific Th cells.

The TCR-M cells activated in the MI context displayed a unique gene expression profile enriched with growth factors and prohealing mediators, but not with cytokines related to the classical Th cell subsets. A list of the top 100 regulated transcripts is provided in Supplemental Figure 2, and the top 30 regulated genes are shown in Figure 4C. Enrichment pathway analyses indicated that transcripts related to $\mathrm{T}$ cell trafficking within LNs (sphingosine 1/ phosphate pathway), the TCR signaling cascade, and responses to hypoxic stress were particularly upregulated in TCR-M cells after MI (Figure 4D and Supplemental Table 2, $P<0.05$ ). With regard to the molecular function of TCR-M cells, unsupervised analyses identified macrophage-CSF (M-CSF) receptor binding, chemokine receptor binding, IL-4 receptor binding, TGF- $\beta$ receptor binding, IL-1 receptor binding, and growth factor activity among the top regulated transcription response patterns in TCR-M cells 
A
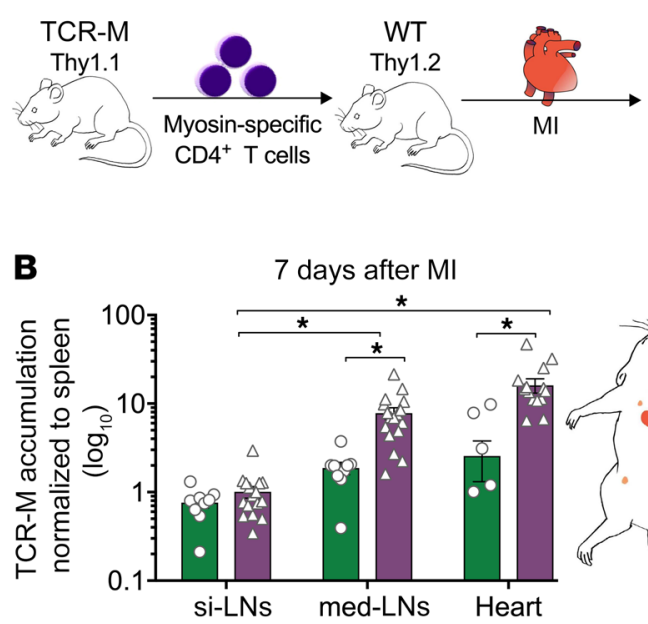

D

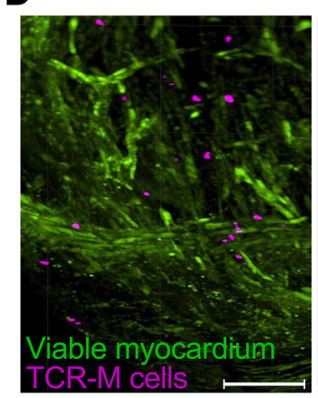

E

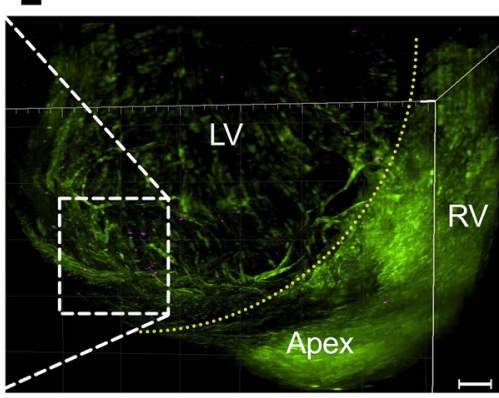

G
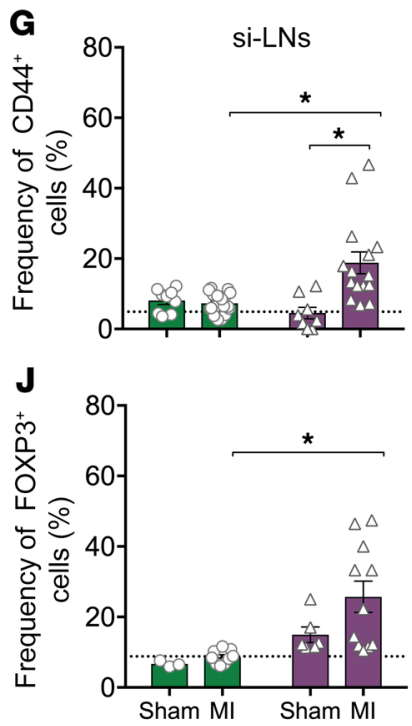

C

$\mathbf{H}_{80}$

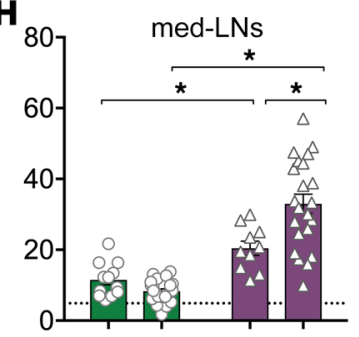

K

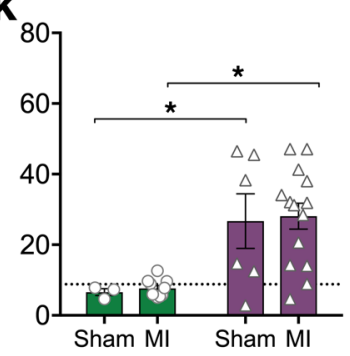

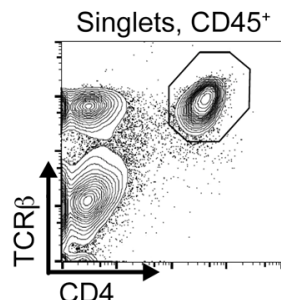
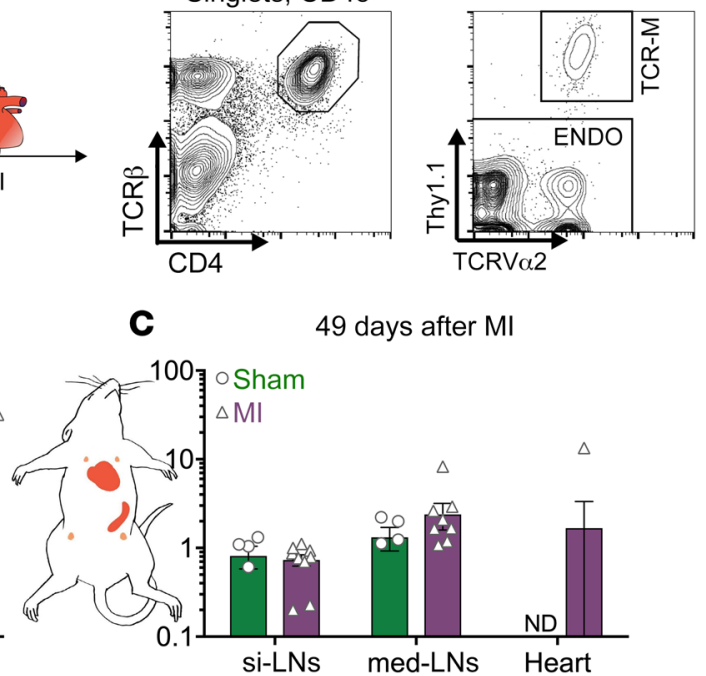

$\mathbf{F}$

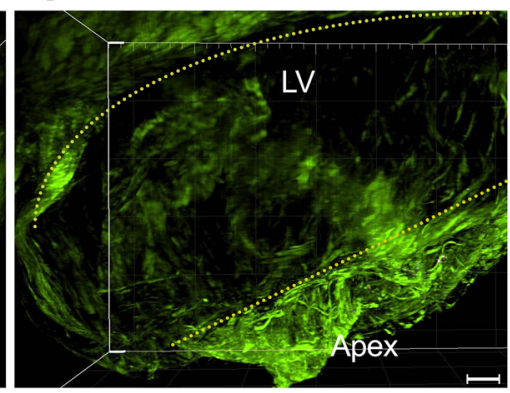

I

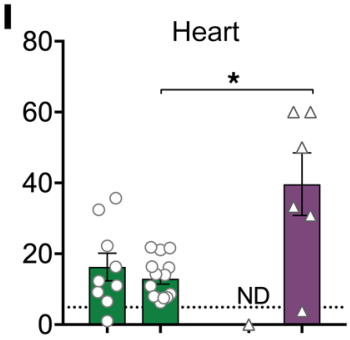

$\mathbf{L}$

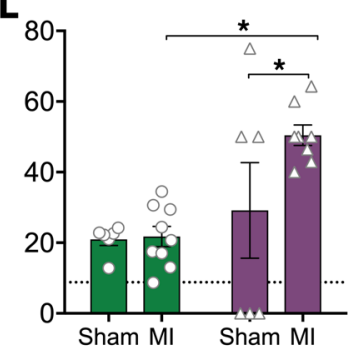

Figure 2. TCR-M cells selectively accumulate in the infarcted heart. (A) Experimental design and gating strategy. Thy1.1 TCR-M cells were transferred into Thy1.2 WT recipients prior to MI or sham operations. The contour plots are representative of the med-LNs 7 days after MI. The frequencies of TCR-M cells found in the si-LNs, med-LNs (heart-draining), and heart were assessed on (B) day 7 and (C) day 49 after MI. The accumulation index refers to the spleen-normalized frequencies. (D and $\mathbf{F}$ ) 3D reconstruction of infarcted hearts (original magnification, $\times 5)$ on day 7 (D and $\mathbf{E}$ ) and day 49 (F) after MI. The morphological information was obtained from the autofluorescence levels acquired in the green channel. The viable myocardium appears in bright green, and the necrotic myocardium appears in dark green. Scale bars: $300 \mu \mathrm{m}$. TCR-M cells (Thy1.1+) appear in magenta, and the yellow dotted lines indicate the infarct borders. (G-L) Frequency of CD44+ cells (G-I) and FOXP3+ cells (J-L) in the ENDO and TCR-M compartments harvested from different sites on day 7 after MI. The dotted lines indicate the baseline frequencies (pre-transfer) of CD44 ${ }^{+}$and $\mathrm{FOXP3}^{+}$among TCR-M cells. The graphs display the group mean values (bars), the SEM, and the distribution of each individual value. (B and C) The green and magenta bars represent sham-operated and infarcted mice, respectively. (G-L) The green and magenta bars represent endogenous $\mathrm{CD} 4^{+} \mathrm{T}$ cells and TCR-M cells, respectively. The data were acquired in at least 2 independent experiments; MI $(n=7-23$ mice) and sham ( $n=3-12$ mice). ${ }^{*} P$ $<0.05$, by 2 -way ANOVA followed by Sidak's multiple comparisons test. LV, left ventricle; RV, right ventricle; ND, not determined. that were activated after MI (Figure 4D, Supplemental Figure 3, and Supplemental Table 3, $P<0.05$ ).

Notably, we found that most of the genes related to classically polarized Th cells (e.g., Ifng, Tnf, Il13, and Il17) were not differentially expressed in TCR-M cells activated after MI (Supplemental Figure 3). Notwithstanding this finding, in response to MI, TCR-M, but not ENDO, cells significantly upregulated a unique set of growth factors and prohealing mediators, such as Csf1, Angptl2, Grn, Tgfb3, and Fgf2 (Figure 4E, $P<0.05$ ). Moreover, TCR-M cells that were stimulated after MI selectively showed unregulated expression levels of inhibitory checkpoint receptors such as Tigit, Pdcd1, Ctla4, and Btla (Figure 4E, $P<0.05$ ). Moreover, these cells were particularly enriched with transcripts related to extracellular purinergic metabolism (Cd38, Nt5e, and Entpd1) and signaling (P2rx4 and P2rx7) (Figure 4E). Taken together, transcriptome profiling revealed that the MI context imposes an inhibitory tonus on polyclonal ENDO cells but favors the development of heartspecific $\mathrm{T}$ cells with a distinct gene expression profile. 


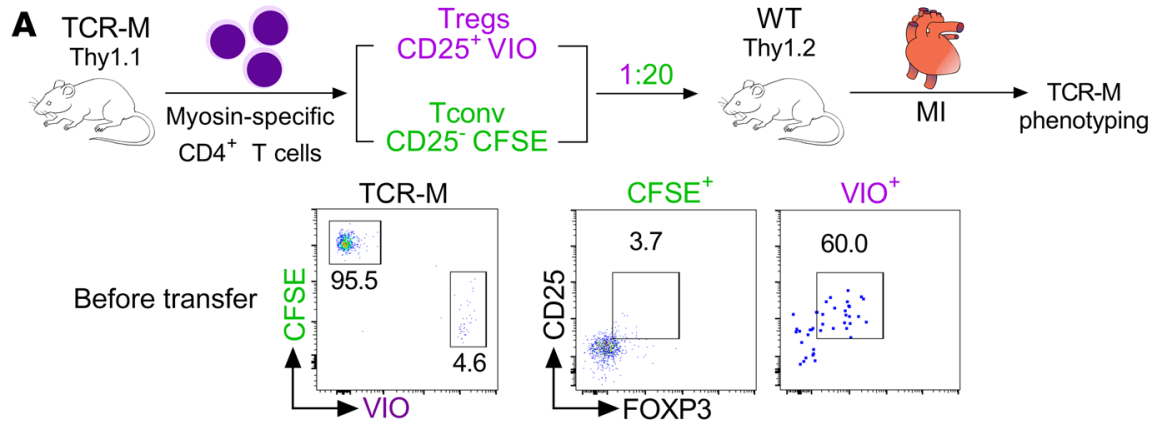

B
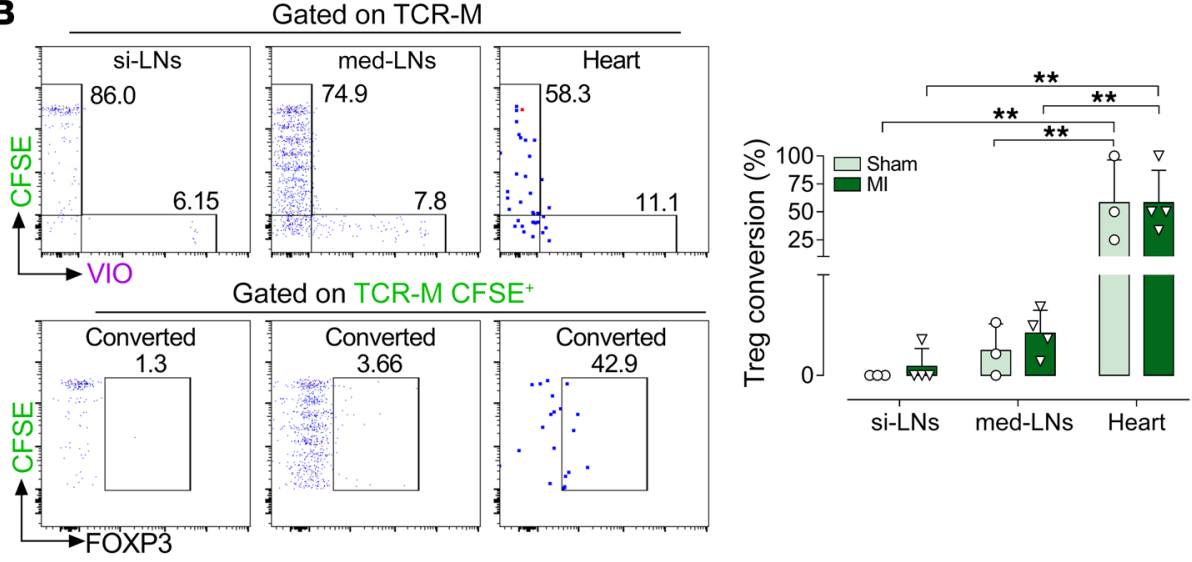

C
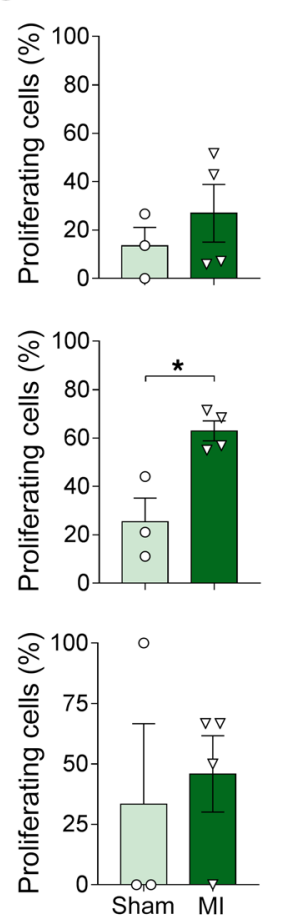

Gated on TCR-M CFSE+
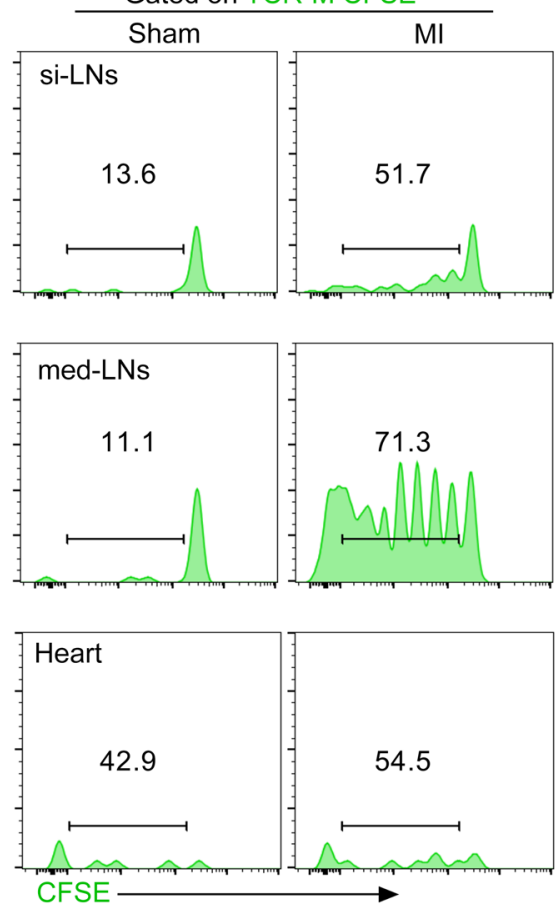

D Gated on TCR-M VIO+
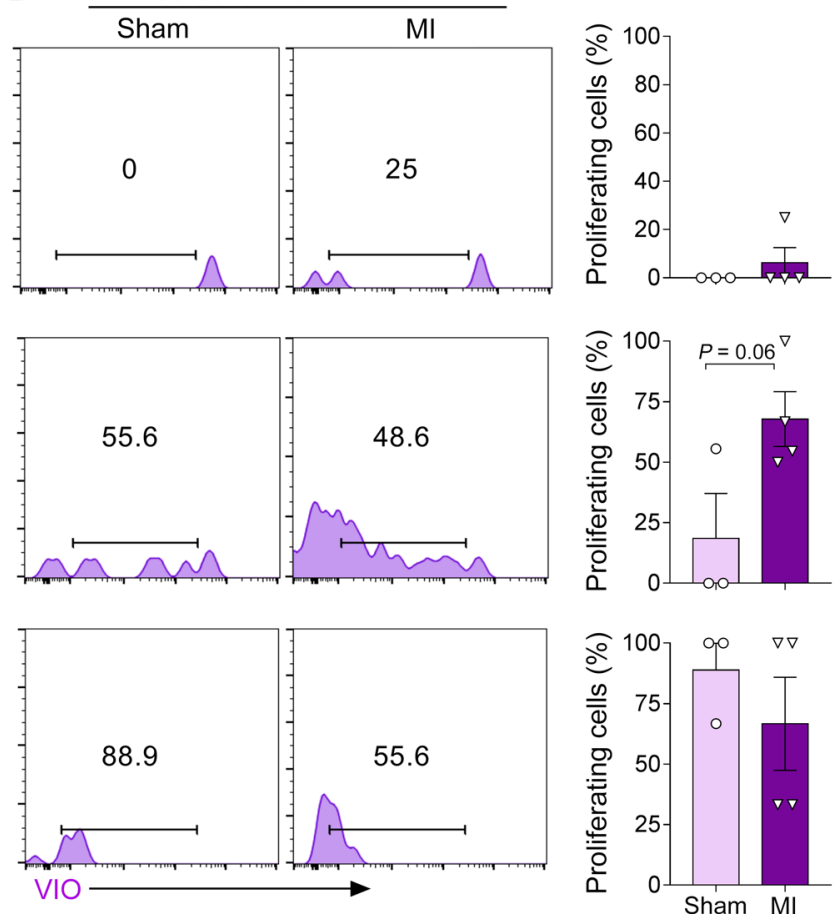

Figure 3. In vivo TCR-M conversion to FOXP3+ Tregs. (A) Experimental design and gating strategy. Before adoptive transfer, Thy1.1+ TCR-M cells were enriched for Tconv cells (CD25-) and Tregs (CD25+) through magnetic cell sorting and labeled with distinct, subset-specific cell-tracer dyes (CFSE and VIO, respectively). Treg and Tconv TCR-M cell populations were mixed at a 1:20 ratio (resembling the physiological condition) and then transferred into Thy1.2 WT recipients 1 day before MI or sham operation. The flow cytometric plots depict the pre-transfer levels of FOXP3 in each compartment. (B) Analysis of Treg conversion from TCR-M cells in si-LNs, med-LNs, and heart tissues from mice 5 days after MI. Converted Tregs are defined as Thy1.1+CFSE ${ }^{+}$cells that acquired FOXP3 expression after MI, as the CFSE ${ }^{+}$cells were FOXP3 ${ }^{-}$prior to cell transfer. (C and D) Proliferation of Tconv TCR-M cells (Thy1.1+CFSE ${ }^{+}$FOXP3-) and Treg TCR-M cells (Thy1.1+VIO+FOXP3 ${ }^{+}$) was assessed through the dilution of CFSE and VIO dyes, respectively. Histograms show the dilution of intracellular fluorescent tracers (CFSE or VIO), indicating the frequency of proliferating cells in each compartment analyzed. ${ }^{*} P<0.01$, by 2 -way ANOVA followed by Tukey's multiple comparisons test (B); ${ }^{*} P<0.05$ and $P=0.06$, by 2-tailed, unpaired $t$ test (C and $\left.\mathbf{D}\right)$. 
A

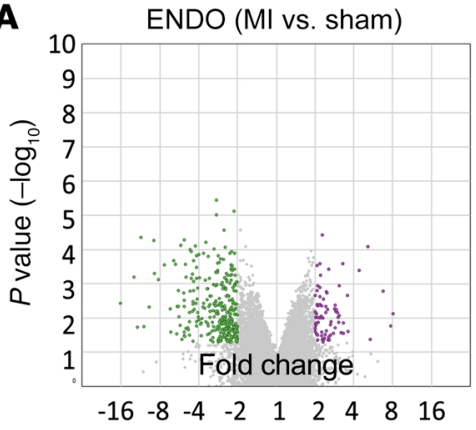

TCR-M (MI vs. sham)

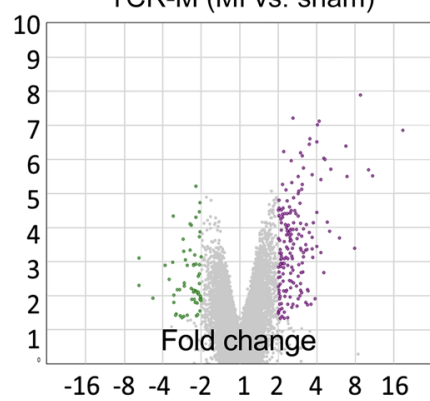

B

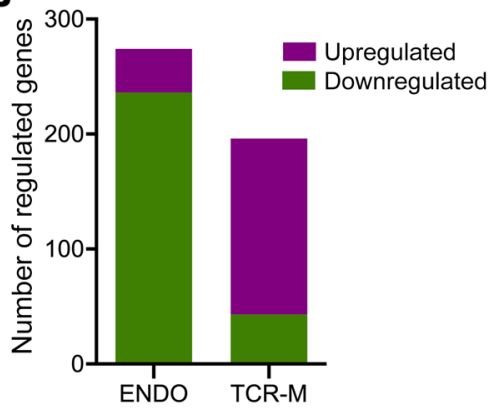

C

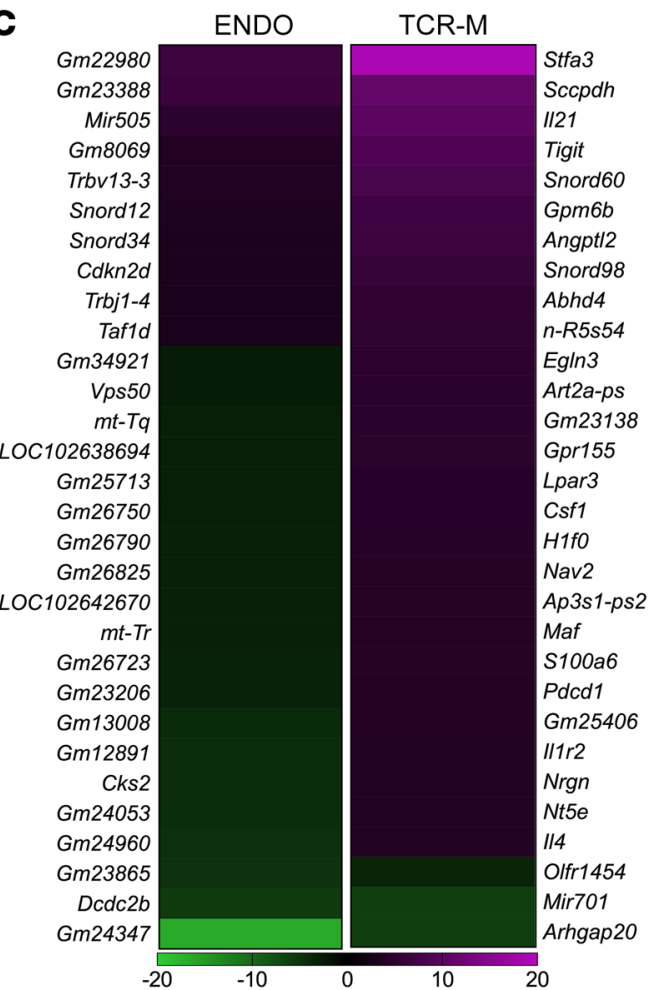

D

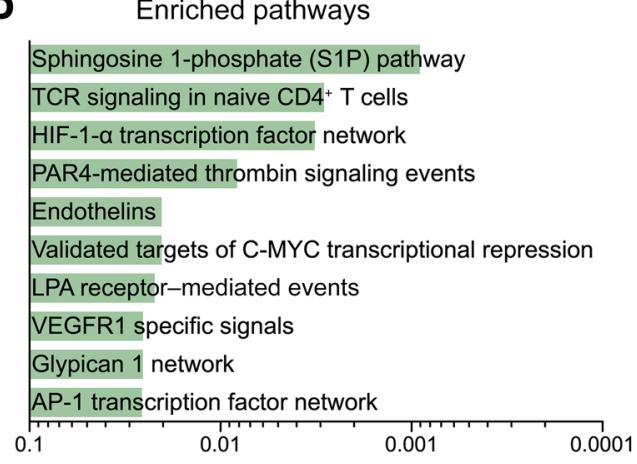

Molecular function

\begin{tabular}{l}
\hline M-CSF receptor binding \\
Chemokine receptor binding \\
IL-4 receptor binding \\
TGF- $\beta$ receptor binding \\
G-protein $\beta / Y$-subunit complex binding \\
IL-1 receptor activity \\
Opioid receptor binding \\
Growth factor activity \\
PI3K activity \\
PTyr phosphatase activity \\
$0.1 \quad 0.11,0.001$ \\
$\quad P$ value $\left(-\log _{10}\right)$
\end{tabular}

E Checkpoint receptors

Tissue repair

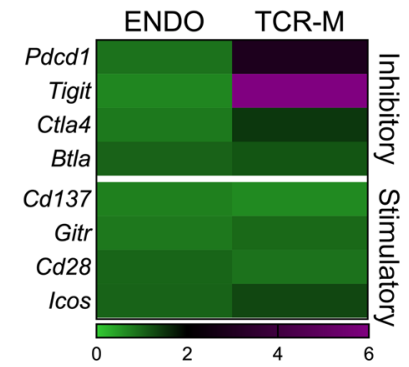

ENDO

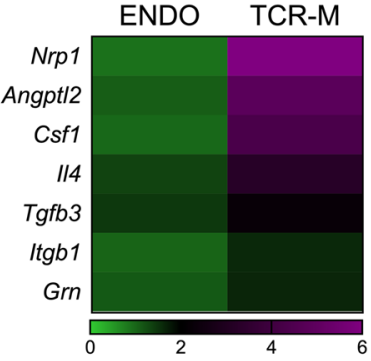

Purinergic metabolism

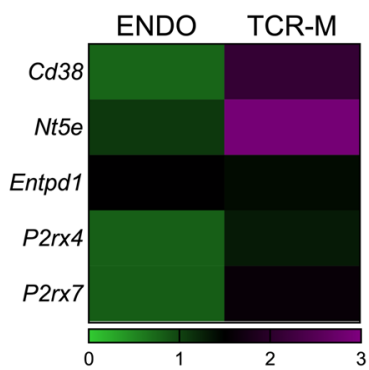

Figure 4. TCR-M cells activated in the MI context acquire a nonclassical gene expression signature enriched with prohealing factors. Adoptively transferred TCR-M cells (defined as CD4+TCR $\beta^{+}$Thy $1.1^{+} T C V \alpha 2^{+}$singlets) and polyclonal endogenous CD4 $4^{+}$ENDO cells (defined as CD4+TCR $\beta^{+}$Thy1.1 $1^{-}$singlets) were sorted from the med-LNs of infarcted and sham-operated mice 7 days after $\mathrm{MI}$ and used for downstream gene expression profiling. (A) Volcano plots comparing the gene expression levels of ENDO and TCR-M cells after MI or sham operation. The repressed and induced genes ( \pm 2 -fold change, $P<0.05$ ) are highlighted in green and purple, respectively. (B) Total number of up- and downregulated genes in each T cell subset. (C) Top 30 differentially expressed genes (MI vs. sham, $P<0.05$ ) in each T cell subset. (D) Unsupervised pathway enrichment analyses and gene clustering according to molecular function (TCR-M subset). The bar lengths indicate the adjusted $P$ values (Fisher's exact test). (E) Normalized relative expression levels (MI vs. sham) of specific gene sets related to T cell activation (checkpoint receptors), tissue repair, and purinergic metabolism. The color scale represents the normalized gene expression levels (MI vs. sham) in ENDO and TCR-M cells. Data for MI $(n=5)$ and sham $(n=3)$ were acquired from 1 experiment. 
Heart-specific CD $4^{+} T$ cells activated in the MI context exert cardioprotective effects. Next, we evaluated the post-MI inflammation and repair processes in mice receiving adoptive transfer of MYHCA- or OVA-specific Th cells (TCR-M and DO11.10 cells, respectively) (Figure 5A). We used OVA-specific $\mathrm{T}$ cells as a control, because OVA is not an autoantigen in mice and hence irrelevant in the MI context. In these experiments, we designated DO11.10 mice as the recipients (35) for the sake of excluding any possible effects of endogenous antigen-specific Th cells. The specificity of the transferred $\mathrm{CD} 4{ }^{+} \mathrm{T}$ cells had no impact on infarct size (Figure 5B, day 7 after MI), heart-to-body weight ratio, wet and dry lung-to-body weight ratio, or absolute numbers of cardiac leukocytes (Supplemental Figure 4, A-C, day 5 after MI). However, echocardiographic analyses performed on day 7 after MI revealed that hearts enriched with autoreactive $\mathrm{CD} 4^{+} \mathrm{T}$ cells had a better-preserved fractional area change (FAC), which serves as a readout for improved systolic function (Figure $5 C, P<0.05$ ). We obtained similar results for the end-systolic area (ESA) measurement (Figure 5D, $P=0.074$ ), but not for the end-diastolic area (EDA) measurement (Figure 5E). Mechanistically, we found that the adoptive transfer of TCR-M cells promoted an increased accumulation of profibrotic MHC-II ${ }^{\text {hi CCR }} 2^{\text {lo }}$ macrophages (36) on day 5 after MI (Figure 5F and Supplemental Figure 4E), compared with DO11.10 cell treatment. Histological analyses of the infarct zone revealed a higher collagen content in the scars of TCR-M-treated mice, as quantified by Picrosirius red staining and collagen III immunofluorescence staining (Figure 5, G and $\mathrm{H}, \mathrm{P}<0.05$ ).

To complement this approach, we also transferred WT lymphocytes purified from the med-LNs of sham versus infarcted mice into lymphocyte-deficient animals and then monitored cardiac function in the recipients (Supplemental Figure 5). These experiments were performed because previous studies in the context of autoimmune myocarditis have established that the transfer of $\mathrm{T}$ cells from diseased mice can produce cardiac injury in unchallenged recipients (34). In contrast to the autoimmune myocarditis context, we observed no signs of myocardial injury mediated by the lymphocytes obtained from infarcted donors (Supplemental Figure $5, \mathrm{~B}-\mathrm{E})$. These findings further support the notion that post-MI T cell autoimmunity is not pathogenic during the early healing phase.

$C D 4^{+} \mathrm{T}$ cells infiltrating the murine infarcted myocardium exhibit a distinct repertoire signature. After showing that $\mathrm{T}$ cells specific for a prototypic myocardial antigen promote myocardial healing via an adoptive transfer approach, we aimed to further confirm whether the endogenous $\mathrm{T}$ cell response is autoantigen dependent. The fundamental role of $\mathrm{T}$ cells in adaptive immunity relies mainly on a diverse repertoire of TCR $\alpha$ and $-\beta$ chains that display unique antigen specificities. Thus, we performed high-throughput sequencing of the TCR $\beta$ complementary determining region 3 (CDR3 $\beta$ ) on FACS-purified CD4 ${ }^{+} \mathrm{T}$ cells from the heart and med-LNs of infarcted and sham-operated mice (Figure 6A and Supplemental Figure 6A) as an unbiased approach to screen for MI-related TCR signatures. As shown in Figure 6, B-D, the $\mathrm{CD} 4^{+} \mathrm{T}$ cells infiltrating the infarcted myocardium exhibited a skewed repertoire dominated by a restricted set of TCR sequences. In the tree maps presented in Figure 6B, each colored spot represents a unique TCR $\beta$ CDR3 sequence (a $\mathrm{T}$ cell clone), and the size of each spot denotes its relative frequency. The unevenness of the spots observed in the cardiac
T cell samples and the high frequencies found for some sequences (37) indicated a clonal expansion of these cells. The Gini inequality coefficient was further calculated as an indicator of the repertoire evenness and clonal dominance (Figure 6C and Supplemental Figure 6B): a Gini coefficient value of 0 denotes an even repertoire, in which every sequence is present in equal abundance, whereas a value of 1 indicates that the repertoire is dominated by a single sequence $(38,39)$. Notably, the cardiac T cells had the highest Gini scores among all the groups (Figure 6C, $P<0.05$ ), which further indicates that the post-MI $\mathrm{T}$ cell responses were marked by oligoclonal expansions. Because the pool of $\mathrm{T}$ cells found in tissues was considerably smaller than that in LNs, we also assessed the Gini coefficient for a subsampling of equal size in each group (Supplemental Figure 6B). The observations remained unaltered also under these conditions. Heart T cells also had a much less diverse repertoire than that found in LNs, as assessed using Simpson's diversity index (Figure 6D and Supplemental Figure 6B). Most strikingly, T cells infiltrating the infarcted myocardium exhibited a unique repertoire signature, as evidenced by several factors. First, the MI-related cardiac TCR repertoire was marked by a particular usage of variable (TRBV) and joining (TRBJ) gene segments (Figure 6, E and F, and Supplemental Figure 6E). In particular, we found that TRBV19 was significantly expanded in MI-related cardiac $\mathrm{T}$ cells compared with its usage in the med-LNs, whereas the usage of TRBV5 was significantly reduced (Figure 6E, $P<0.05$ ). Second, the CDR3 length distribution of TRBV19 sequences was skewed in the heart samples, whereas a Gaussian distribution was found in the LN samples (Supplemental Figure 6D). Because V-J recombination is a random process, the length distribution of natural repertoires tends to have a Gaussian distribution. Therefore, biases in the distribution of TCR $\beta$ CDR3 lengths are indicative of clonal expansions (40). Third, MI-related T cells had a unique pool of sequences exhibiting minimal overlap with all the other samples investigated in our study (Figure 6G and Supplemental Figure 6F).

The identity of cognate antigens could be directly extrapolated from the TCR sequence data. Therefore, we also assessed the TCR repertoire of $\mathrm{CD}^{+} \mathrm{T}$ cells purified from the axillary LNs (ax-LNs) of mice that were subcutaneously immunized with cardiac myosin and heart extract (in adjuvants), because these repertoires were expected to be enriched in heart-specific sequences upon specific immunization. As a control, we also assessed the ax-LN TCR repertoire in mice that received subcutaneous administration of the adjuvant alone. The high degree of clonality exhibited by the cardiac $\mathrm{T}$ cell pool remained statistically significant compared with that found for all ax-LN repertoires, even those from immunized mice (Supplemental Figure 7, A-E). Notably, the Th cell repertoire found in the ax-LNs of mice that received heart extract immunization also showed an increased TRBV19/TRBV5 ratio, similar to that found for cardiac $\mathrm{T}$ cells and in sharp contrast to that found for the control ax-LNs (Supplemental Figure 7, F and G). Notwithstanding, the comparison of the TCR $\beta$ CDR3 sequences in the infarcted and immunized groups revealed no significant repertoire overlap. To a great extent, this result might be related to the fact that the cardiac $\mathrm{T}$ cell repertoire is largely composed of private sequences (i.e., no CDR3 repertoire overlap between individual cardiac EMI samples).

Taken together, the results from this unbiased screening approach revealed that the $\mathrm{CD} 4^{+} \mathrm{T}$ cells infiltrating the infarcted 
A

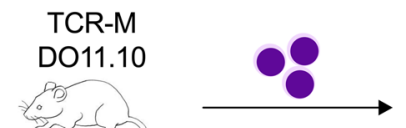

MYHCANOVA-specific $\mathrm{CD} 4^{+} \mathrm{T}$ cells
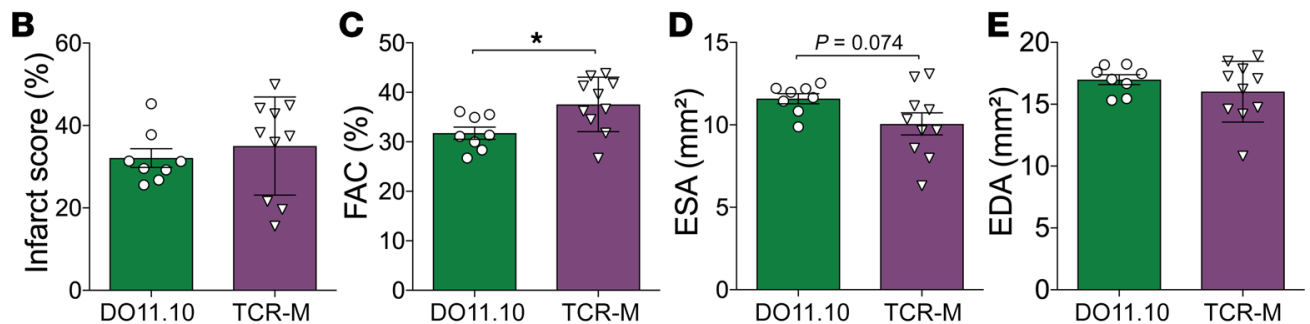

$\mathbf{F}$
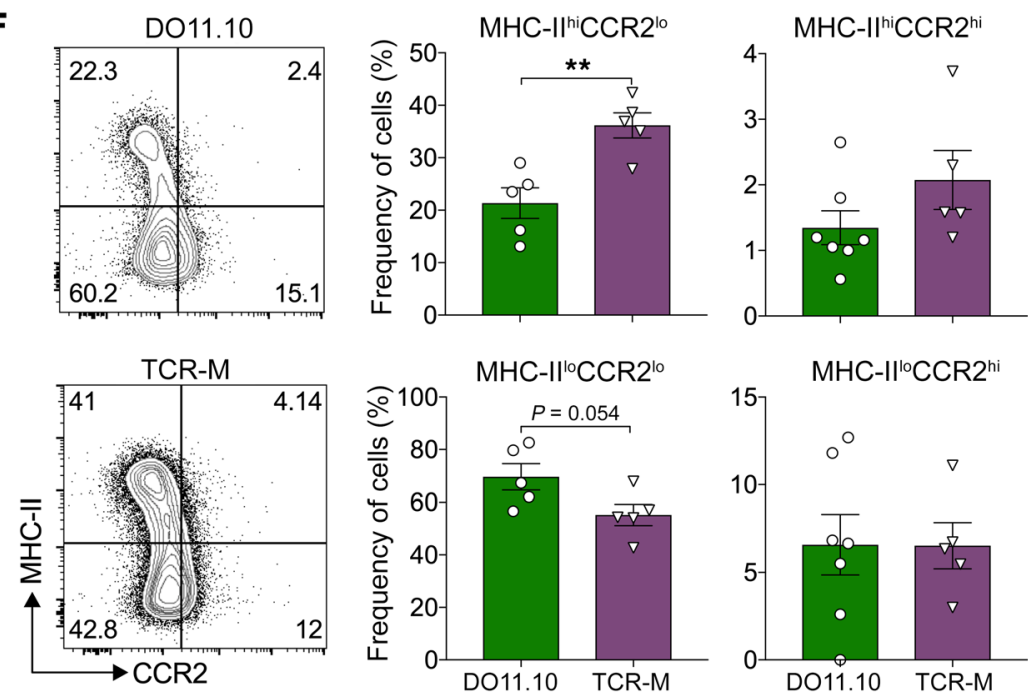

G

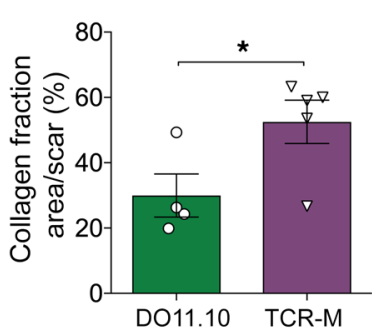

H

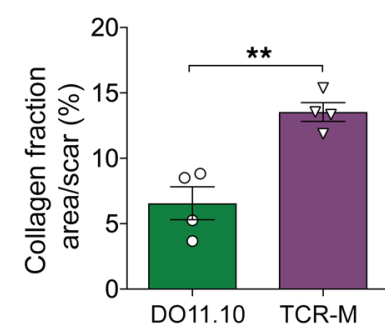

MHC-III $\mathrm{CCR} 2{ }^{10}$
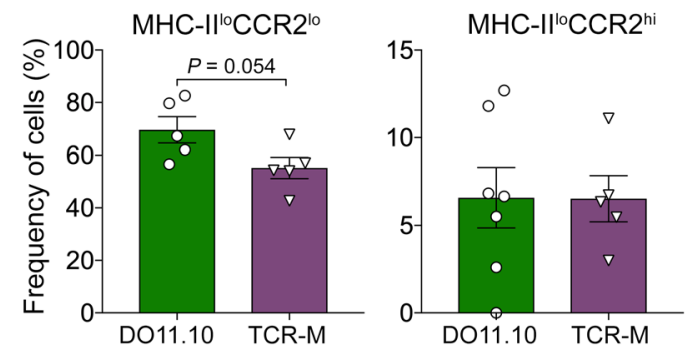

Remote
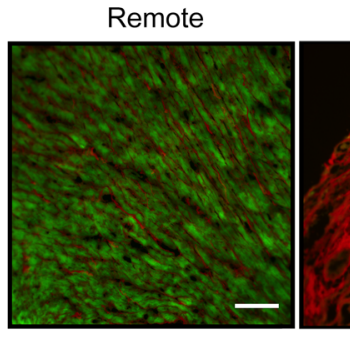

DO11.10

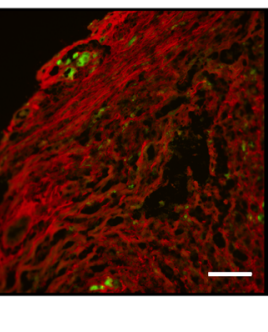

TCR-M
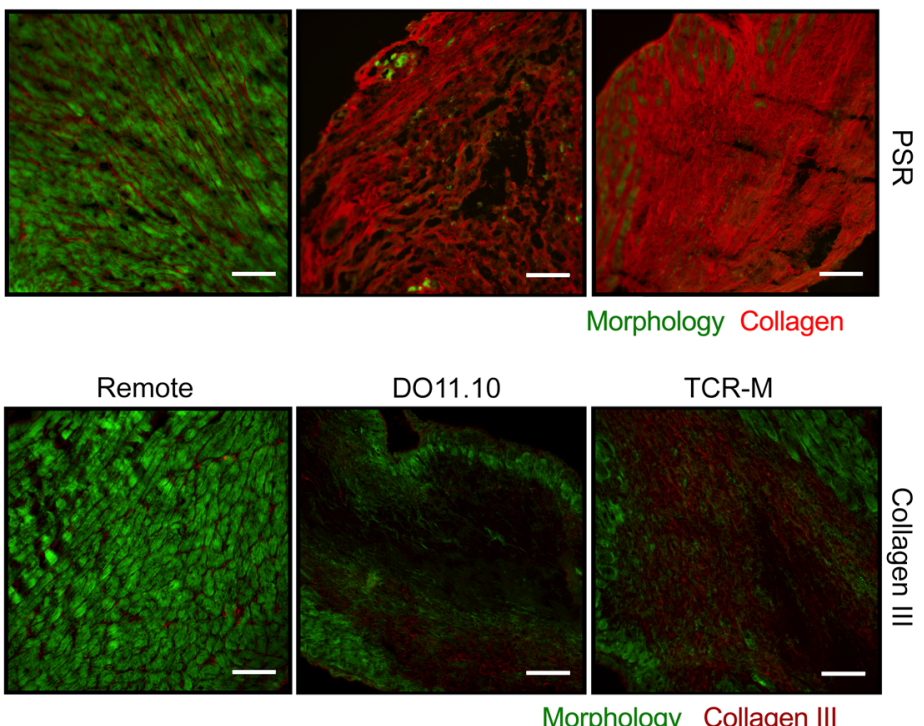

TCR-M

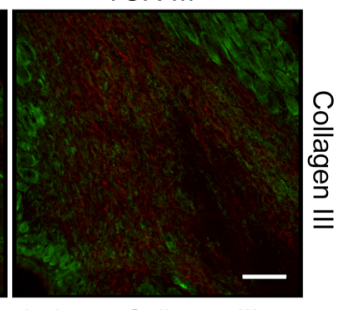

DO11.10

Remote

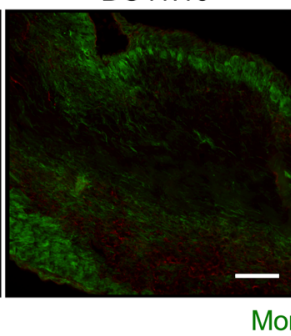

Figure 5. Heart-specific CD4 ${ }^{+} \mathrm{T}$ cells activated in the MI context exert cardioprotective effects.

(A) Experimental design: TCR-M cells (specific for the cardiac antigen MYCA $\left.{ }_{614-629}\right)$ and D011.10 cells (specific for the irrelevant antigen $\mathrm{OVA}_{\text {323-339 }}$ ) were adoptively transferred into D011.10 mice prior to $\mathrm{Ml}$ or sham operation, and the cardiac outcomes were monitored at the peak of the healing phase (day 7). (B) The infarct size and long parasternal axis (apex-aortic valve) were assessed by echocardiography. The (C) FAC, (D) ESA, and (E) EDA at the mid-papillary level were assessed by echocardiography. (F) Cardiac macrophages, defined as CD45+CD11b+Ly6C-CD64+ singlets, were stratified into 4 major subsets according to CCR2 and MHC-II expression, and the effects in D011.10 versus TCR-M cells on each subset's distribution were assessed (day 5). (C) The collagen area in scar tissue 5 days after MI in D011.10- and TCR-Mtransferred mice was quantified by Picrosirius red staining (PSR). (H) Immunofluorescence of collagen III and its quantification in scar tissue 5 days after MI in D011.10and TCR-M-transferred mice. Scale bars: $100 \mu \mathrm{M}$. The bar graphs display the group mean values (bars), the SEM, and the distribution of each individual sample. Statistical significance was determined by 2-tailed, unpaired $t$ test. ${ }^{*} P<0.05$ and ${ }^{*} P<0.01$. The data from TCR-M $(n=5-10)$ and D011.10 $(n=$ $7-9$ ) recipients were acquired in 3 experiments. myocardium had a distinct $\mathrm{T}$ cell repertoire signature dominated by a limited set of expanded clones. These characteristics are hallmarks of antigen-driven responses.

Evidence for a heart/T cell axis in MI patients. We subsequently sought to assess the existence of a putative heart/T cell axis in MI patients. Our EMI findings showed that the most important T cells are either in the heart or the med-LNs (heart-draining LNs) rather than in the periphery. To validate these findings, we assessed the presence of $\mathrm{FOXP3}^{+}$and $\mathrm{CD}^{+}$cells in cardiac autopsy samples. Twenty-three patients were diagnosed at autopsy with having had a recent MI in the left ventricle. These MI patients were further categorized into 3 distinct reaction phases. The early phase $(\sim 3-$ 
A

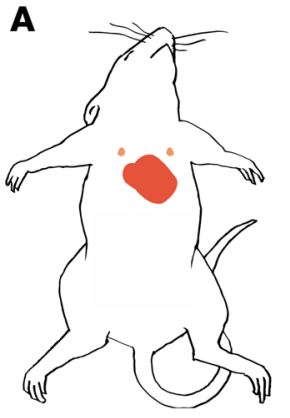

C

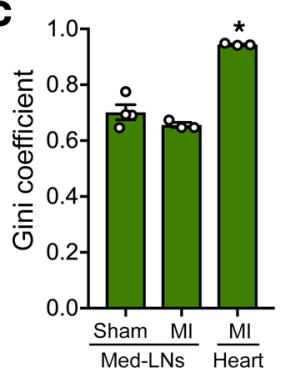

B

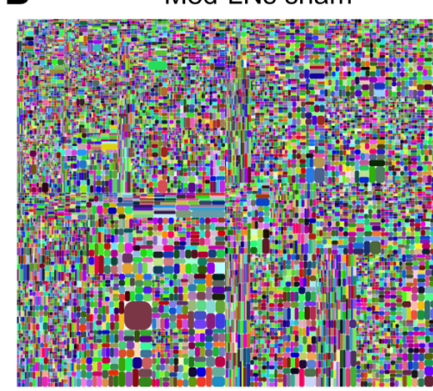

D

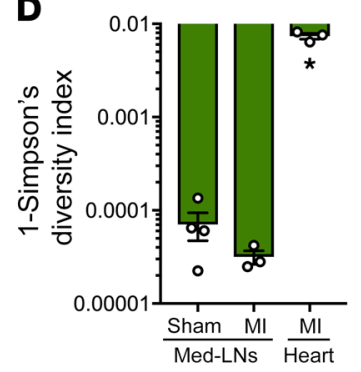

Med-LNs MI

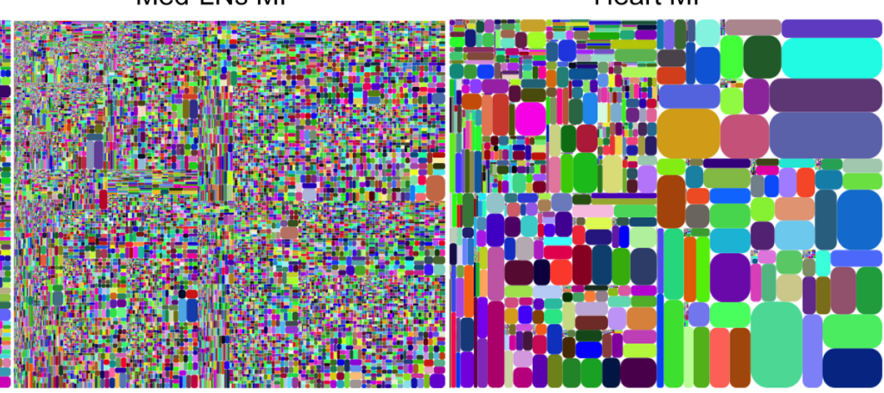

$\mathbf{E}$

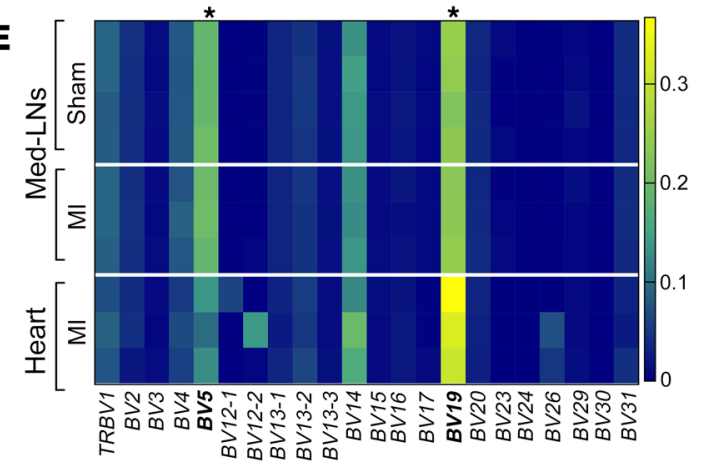

$\mathbf{F}$

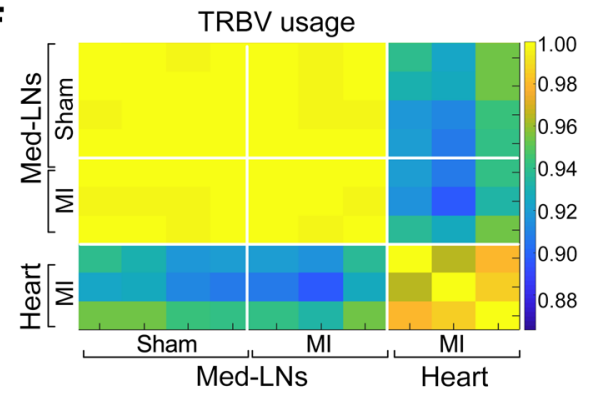

G

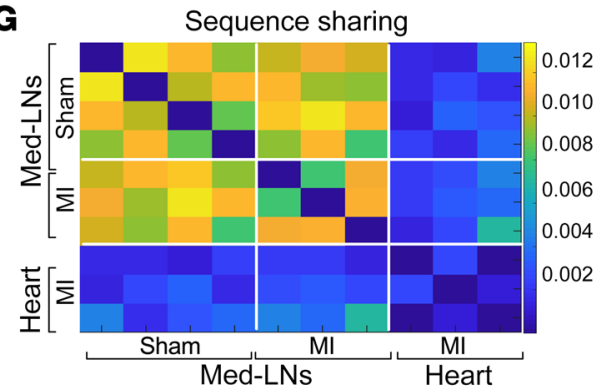

Figure 6. CD4 ${ }^{+} \mathrm{T}$ cells infiltrating the murine infarcted myocardium are clonally expanded and exhibit a unique repertoire signature. (A) Th cells from the heart and med-LNs of infarcted and sham-operated mice (day 7) were purified by FACS. (B) Tree maps of representative repertoires from each group. Each spot represents a unique TRBV-CDR3 recombination, and the size of each spot denotes its relative frequency. The unevenness of the spots indicates clonal expansions. (C) Repertoire evenness was assessed by the Gini coefficient. (D) Repertoire diversity was assessed on the basis of 1-Simpson's diversity index. (E) Heatmap depicting the TRBV gene usage in each group. The asterisks at the top of the graph indicate a statistically significant difference $(P<$ 0.05 ) between the cardiac and LN repertoires, as determined by 2-way ANOVA followed by Tukey's multiple comparisons test. (F) Correlation among the frequencies of the TRBV gene segment between any 2 given samples. (G) Heatmap showing the degree of TRBV CDR3 sequence sharing (Jaccard index) between any 2 given samples. The bar graphs in $\mathbf{C}$ and $\mathbf{D}$ display the group mean values (bars), the SEM of 3 to 4 samples per group, and the distribution of each individual value. Statistical significance for $\mathbf{C}$ and $\mathbf{D}$ was determined by 1-way ANOVA followed by Dunnett's multiple comparisons test. ${ }^{*} P<0.05$ compared with all the other groups.

12 hours after MI) was marked by lactate dehydrogenase (LDH) decoloration without neutrophilic cell infiltration $(n=6)$. In the inflammatory phase (12 hours to 5 days after MI), neutrophilic granulocytes were particularly observed in the infarcted myocardium $(n=9)$, whereas in the proliferation phase (5-14 days after MI), the formation of granulation tissue was obvious $(n=8)$. Nine patients without pathological evidence of MI-related death were included as controls. A description of the patients in whom cardiac autopsies were performed is provided in Supplemental Table 4. As shown in Figure 7, A-E, we found $\mathrm{CD}^{+}$and $\mathrm{FOXP3}^{+}$cells in the infarcted hearts, and their expression levels peaked during the proliferative phase of wound healing $(P<0.05)$. Neither $\mathrm{CD} 4^{+}$nor $\mathrm{FOXP}^{+}$cells were found in the control hearts.
Several mouse studies have shown that $\mathrm{T}$ cells infiltrating the injured myocardium are activated in and originate from the medLNs $(16,17,25)$, suggesting that assessing med-LN morphology and cellularity in patients with MI could also yield clinically relevant information. Thus, we acquired thoracic $\mathrm{CT}$ images from MI patients to assess med-LN morphology. Moreover, we combined CT with PET imaging using a radiolabeled CXCR4 ligand ( $\left[{ }^{68} \mathrm{Ga}\right]$ pentixafor). CXCR4 is a chemokine receptor that is constitutively expressed at high levels on $\mathrm{T}$ cells and regulates their migration along gradients of the chemokine CXCL12 (41). CXCR4 is also expressed on some monocyte subsets (42), on B cell subsets (43), and on hematopoietic stem cells (44), which are present at only small frequencies within LNs. A flow cytometric analysis 
A Early phase
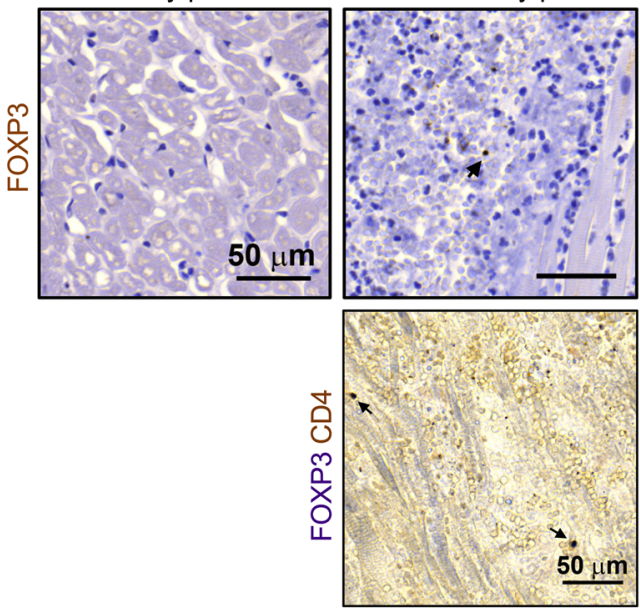

C
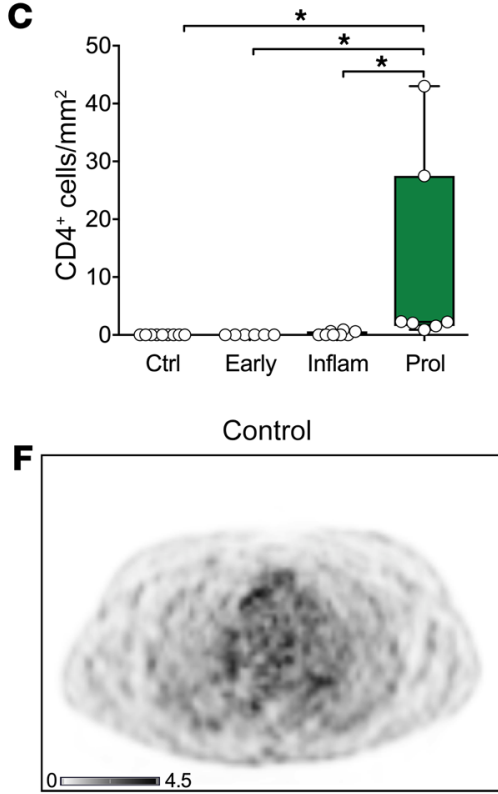

H

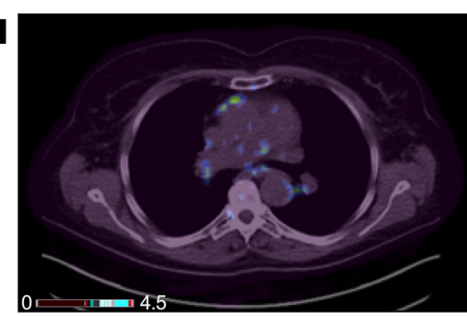

D

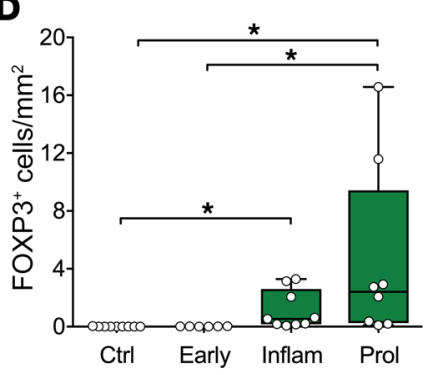

B

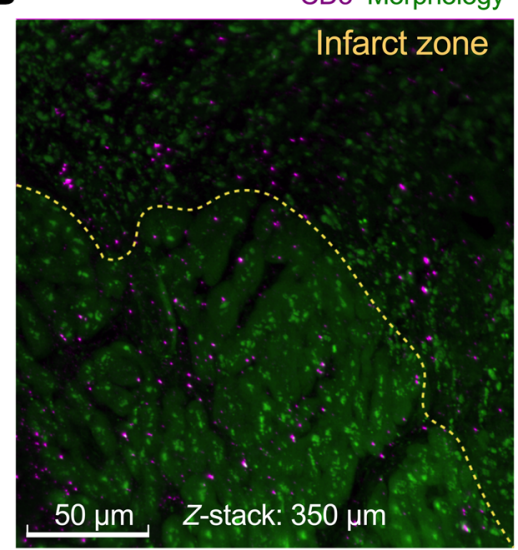

E

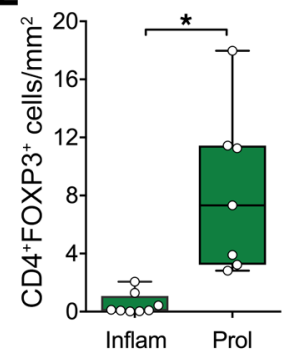

CD3 Morphology

$50 \mu$
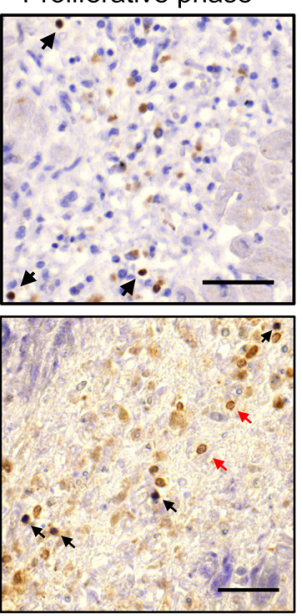

G

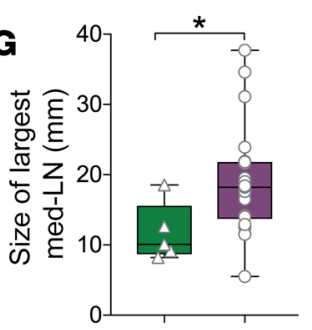

I

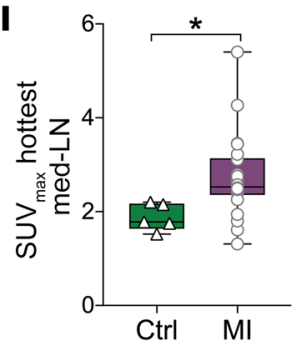

Figure 7. A heart/T cell axis in MI patients. (A-E) Histological analyses of cardiac autopsy samples revealed that Th cells also accumulated in the human infarcted myocardium, particularly during the proliferative (wound-healing) phase. (A) Representative IHC micrographs show the presence of FOXP3 ${ }^{+}$ (black arrows) and CD4+ (red arrows) cells in cardiac tissues. Scale bars: $50 \mu \mathrm{m}$. (B) LSFM showing the presence of CD3 ${ }^{+} \mathrm{T}$ cells in the infarcted human myocardium. Z-stack: $350 \mu \mathrm{m}$. (C-E) Numbers of $\mathrm{CD}^{+}, \mathrm{FOXP3}^{+}$, and $\mathrm{CD} 4^{+} \mathrm{FOXP3}^{+}$cells $/ \mathrm{mm}^{2}$ in each phase after MI (early, inflammatory, proliferative). The whiskers represent the percentile range, with the medians \pm Cls from 6 to 9 samples per group. ${ }^{*} P<0.05$, by nonparametric Kruskal-Wallis test corrected for multiple comparisons using Dunn's test. (F-H) Transaxial slices of (F) PET and (H) fused PET/CT showing increased CXCR4 expression in med-LNs after MI (arrows) and in the infarcted myocardium (inset). Both the (G) med-LN sizes and (I) CXCR4 expression (as assessed by SUVs) were found to be increased after MI. The data are presented as box plots showing the medians, $25^{\text {th }}-75^{\text {th }}$ percentiles (boxes), and $5^{\text {th }}-95^{\text {th }}$ percentiles (whiskers) of 6 to 9 cardiac autopsy samples, or 26 patients (PET/CT) per group. ${ }^{*} P<0.05$, by Fisher's exact test (C-E) and Welch's $t$ test (G and I). 
of the CXCR4 distribution across different leukocyte subsets in mice confirmed that $\mathrm{T}$ cells accounted for more than $90 \%$ of the $\mathrm{CXCR}^{+}$cells in the med-LNs but less than $15 \%$ of the CXCR4 ${ }^{+}$ cells in the heart (Supplemental Figure 8). We therefore used a noninvasive CXCR4 monitoring strategy to obtain a readout for $\mathrm{T}$ cellularity in heart-draining LNs. Patients with nonmalignant Conn's adenoma who had undergone $\left[{ }^{68} \mathrm{Ga}\right]$ pentixafor PET/CT for a diagnostic workup were included as controls. Conn's adenomas are aldosterone-producing benign conditions that account for $30 \%$ to $40 \%$ of all cases of primary aldosteronism. Because these adenomas overexpress CXCR4 (45), these patients were subjected to receptor-directed imaging as part of an endocrinological investigation, and none of these patients were found to have elevated serum levels of C-reactive protein $(<0.5 \mathrm{mg} / \mathrm{dL})$.

As shown in Figure 7, F and G, the med-LNs were significantly enlarged in MI patients but not in the control group patients (19.1 $\pm 7.6 \mathrm{~mm}$ versus $11.7 \pm 4.2 \mathrm{~mm} ; P<0.05$, measured on day 7 after MI, range, 2-13 days). The patients with MI also had significantly increased CXCR4 expression in the med-LNs compared with the control patients (as derived by $\left[{ }^{68} \mathrm{Ga}\right]$ pentixafor PET/CT; maximum standardized uptake value $\left[\mathrm{SUV}_{\max }\right]$ of $2.7 \pm 0.9$ versus 1.9 $\pm 0.3, P<0.001$, Figure $7, \mathrm{G}-\mathrm{I})$. These findings indicated that the med-LN enlargement observed in MI patients might at least in part be related to local expansion in the T cell compartment. Furthermore, the size of the med-LNs showed a moderate correlation with the infarct volume derived by cardiac magnetic resonance imaging (CMR) $(r=0.45, P=0.05$, Supplemental Figure 9A) and with the levels of serum creatine kinase (CK) after MI (sum of CK, $r=0.57, P<0.01$, Supplemental Figure 9B). The uptake of $\left[{ }^{68} \mathrm{Ga}\right]$ pentixafor in the med-LNs was positively correlated with CXCR4 expression in the infarcted myocardium $(r=0.52, P=0.03$, Supplemental Figure 9C). Moreover, the CXCR4 signal in the medLNs suggested a positive prognosis, with smaller myocardial necrosis volumes in a follow-up CMR imaging study (median 4 months after MI, $r=-0.51, P=0.06$, Supplemental Figure 9D). Taken together, these clinical findings reveal for the first time to our knowledge the existence of a local heart/med-LN/T cell axis in patients with MI and show remarkable similarities with the findings in the framework of EMI.

\section{Discussion}

Emerging concepts in the field of cardiology suggest that the infarcted myocardium could be perceived as a wounded tissue (46), and hence the immunological phenomena underlying myocardial repair have received considerable attention worldwide $(5$, $8,47-51)$. The contribution of innate immune mechanisms to postMI inflammation, healing, and remodeling has been well scrutinized over the past 2 decades $(52,53)$, but the involvement of the adaptive arm of the immune system has only recently been recognized $(8,16,18,20,22,54,55)$. In this study, we assessed global features of the post-MI $\mathrm{T}$ cell repertoire and pinpointed a defined cardiac antigen that is targeted by $\mathrm{CD} 4^{+} \mathrm{T}$ cells after EMI to dissect the bidirectional cardio-immune crosstalk in unprecedented detail. Moreover, through a combination of histological analyses of cardiac tissue samples with noninvasive imaging of the medLNs in MI patients, we provide evidence that such a heart/T cell axis might hold great translational implications.
T cells reportedly influence cardiac injury, repair, and remodeling in various organisms, ranging from fishes to mammals $(16,18-21,56)$. Nevertheless, the question of whether post-MI autoimmune responses exert salutary or detrimental effects has remained debated (54). Independent laboratories have recently reported that $\mathrm{T}$ cells may foster early cardiac healing by modulating macrophage polarization (18), fibroblast activity (20), and extracellular purinergic metabolism (21). However, chronic T cell activation in the myocardium has also been reported to fuel the development of adverse remodeling in models of ischemic and stress overload-induced HF (19, 25-27). Moreover, the reactivity profile of T cells activated in the MI context has not to our knowledge been assessed, and whether these cells target specific antigens released by dying cardiomyocytes or simply respond to the inflammatory milieu remains largely unclear (57).

Here, we found that $\mathrm{T}$ cells infiltrating the infarcted myocardium display a unique repertoire signature marked by a preferential expansion of TCRBV19-expressing clones. Notably, we also observed a similar bias toward the use of TRBV19 gene segments in mice immunized with cardiac antigens. In a previous study, $\mathrm{Hu}$ et al. (58) also observed skewed TRBV19 ${ }^{+} \mathrm{T}$ cell responses in a murine model of cardiac allograft transplantation. Furthermore, Klingenberg et al. (59), Heidecker et al. (60), and Blohm et al. (61) independently reported elevated TCRBV19 expression in coronary thrombi obtained from patients with coronary syndrome, and in cardiac biopsies from patients with autoimmune myocarditis and dilated cardiomyopathy, respectively. Taken together, these findings strongly suggest that TRBV19+ ${ }^{+}$cells might be associated with heart-specific $\mathrm{T}$ cell responses in different organisms, ranging from mice to humans. The causes underlying this remarkable association between specific TR $\beta \mathrm{V}$ usage and cardiotropic immune responses remain unclear, but the converging evidence might encourage future research that will elucidate this point.

In the present study, we identified cardiac myosin (MYHCA) as a dominant cardiac antigen in the MI context and established a defined system for dissecting the role of MYHCA-specific T cell responses in the wounded myocardium in unprecedented detail. MYHCA is a prototypical cardiac antigen for several reasons: it is selectively expressed in the myocardium but not in any other tissue $(31,62)$; it is constitutively processed and presented to $\mathrm{T}$ cells by cardiac antigen-presenting cells (34); in contrast to most tissue-restricted proteins, MYHCA is not expressed by thymic epithelial cells, meaning that central tolerance to this cardiac antigen is absent or impaired (63); and it has been strongly implicated in the pathogenesis of autoimmune myocarditis (33).

In addition to specificity, our data reveal that the myocardial milieu dictates $\mathrm{T}$ cell phenotypic polarization favoring the in situ induction of Treg cells and the development of protective autoimmune responses that benefit tissue repair through the promotion of collagen deposition. The latter findings are in accordance with those obtained in our previous study, which revealed that mice lacking $\mathrm{CD}^{+} \mathrm{T}$ cells have impaired collagen deposition after EMI (16). The detailed mechanism underlying the T cell-mediated myocardial fibrosis process remains elusive, but our findings suggest that it might be dependent on crosstalk with MHC-II ${ }^{\text {hi }}$ CCR $2^{\text {lo }}$ macrophages, which are upregulated in TCR-M-transferred mice. Recent studies have established that MHC-II ${ }^{\text {hi }} \mathrm{CCR} 2^{\mathrm{lo}}$ mac- 
rophages exhibit a transcriptome profile enriched with profibrotic genes, show lower metalloproteinase activity, and are involved in the cardiac fibrosis process in models of diastolic dysfunction (36).

It is important to consider that the role of autoreactive $\mathrm{T}$ cells in MI and HF is likely stage dependent. This is particularly relevant for T cell-mediated cardiac fibrosis. Building a solid and fast scar after MI helps prevent cardiac rupture and infarct expansion, but progressive collagen deposition can also lead to adverse remodeling (7). This might help explain why cardiac $\mathrm{CD}^{+} \mathrm{T}$ cells have been recently implicated in both salutary and pathogenic mechanisms (16-28). Furthermore, it is important to recognize that TCR-M cells induce autoimmune myocarditis in donor mice (33). Together, these findings indicate that the outcome of heart autoimmunity is largely context dependent and reveal an what we believe to be an unprecedented role for the myocardial milieu in the shaping of $\mathrm{T}$ cell phenotypic polarization.

A similar dual role for Th cells in mediating tissue-specific autoimmunity and repair was previously reported by Schwartz et al. (11). These authors observed that the same antigen-specific $\mathrm{CD}^{+} \mathrm{T}$ cells that mediate experimental autoimmune encephalomyelitis can also foster spinal cord repair. Understanding the differences between physiological and pathogenic heart-specific autoimmunity might be a central issue to be scrutinized in future studies in this field.

To the best of our knowledge, we provide the first report of the existence of a heart/med-LN/T cell axis in patients with MI. Moderate infiltration of $\mathrm{CD}^{+}$and $\mathrm{FOXP}^{+}$cells was consistently observed in the infarcted myocardia of mice and humans. The kinetics of cardiac $\mathrm{T}$ cell infiltration showed substantial similarities across species, and the numbers of infiltrating cardiac $\mathrm{T}$ cells peaked at the healing phase. MI patients also show alterations in their med-LN morphology and cellular composition that are similar to those observed in rodents $(16,17,24)$. CT measurements provided valuable information of the med-LN morphology but could also aid the discrimination of alterations due to edema from those due to increased cellularity. Therefore, the critical advantage provided by combined CT/CXCR4 PET tracing is that this approach provides strong evidence for alterations due to increased cellularity, which is a hallmark of adaptive immune responses. We acknowledge that the widespread distribution of CXCR4 expression across different cell types demands careful interpretation of these findings. However, the limited specificity of this target can be compensated by the anatomical specificity of our observations, because lymphocytes account for the vast majority of cells within LNs (64). It might therefore be safe to conclude that the PET/CT approach used in this study could offer a suitable method for monitoring local adaptive immune responses in MI patients. Indeed, a recently published study revealed that CXCR4 plays an important role in controlling T cell trafficking in the context of EMI and thereby influences the healing outcome (65), and these findings provide great mechanistic support for our clinical findings.

The observed correlation between alterations in the med-LNs and the clinical findings (e.g., infarct volume and follow-up cardiac function) reinforces the clinical relevance of this approach and indicates that imaging the heart-draining LNs might provide additional valuable clinical information. This bold step might help overcome the limitations of peripheral blood sampling (only $<2 \%$ of all lymphocytes are typically present in the peripheral blood) (66) and might even allow the tailoring of directed immunotherapeutic interventions in the future.

Cardiologists can rely on good therapeutic and interventional options for the treatment of acute MI and for attenuating the progression of remodeling $(9,10)$, but no tools are currently available for fostering the myocardial healing processes that bridge these 2 conditions. In fact, prospectively validated biomarkers are currently not available for monitoring the quality of ongoing myocardial healing, and thus clinicians cannot identify patients undergoing adverse remodeling until they reach a critical HF stage. Hence, there is an unmet need in the field of cardiology to better understand and eventually modulate the mechanisms underlying the myocardial repair process $(8,67)$. A new discipline at the crossroads between immunology and cardiology has rapidly flourished over the past decade, with the aim of filling these gaps. From the cardiological perspective, this multidisciplinary enterprise might offer new and exciting opportunities for preventing the development of ischemic HF, which is a consequence of poor post-MI healing. From an immunological perspective, this association with cardiology might offer a unique opportunity to better understand the often-neglected aspects of physiological autoimmunity.

\section{Methods}

Detailed descriptions of the experimental methods are provided in the Supplemental Methods.

\section{Study design}

Mouse studies. Our aim was to clarify the functional significance of autoreactive T cell responses to EMI. Therefore, we selected a model of transgenic murine cardiac myosin-specific CD $4^{+} \mathrm{T}$ cells (TCR-M) that were adoptively transferred into $\mathrm{WT}$ and DO11.10 recipient mice prior to the induction of EMI through permanent surgical ligation of the left coronary artery. The recipient DO11.10-treated mice were selected in some experiments because the entire $\mathrm{CD} 4^{+} \mathrm{T}$ cell compartment in these animals is composed of OVA-specific cells (i.e., an irrelevant antigen in the MI context). The distribution and differentiation of endogenous and transferred T cells were analyzed by flow cytometry and transcriptomics of sorted cells. Echocardiography was performed to characterize the clinical phenotype of the animals. To further study the role of endogenous lymphocytes activated in the MI context, bulk med-LN-derived cells were adoptively transferred into naive lymphocyte-deficient Rag1-KO mice. Furthermore, we performed an analysis of the TCR repertoire using a TCR sequencing approach to confirm the clonal expansion of endogenous $\mathrm{T}$ cells in response to MI.

The echocardiographic imaging data were evaluated by a blinded experimenter. The exclusion criteria were set a priori according to the heart rate during echocardiography (heart rate $>450 \mathrm{bpm}$ ), and the FACS cell yields were too low to draw reliable conclusions. Outliers were not excluded from the statistical analyses.

Human studies. The translational relevance of the murine findings obtained in the current study was tested in a clinical setting using histological and imaging approaches. $\mathrm{CD}^{+}$and $\mathrm{FOXP3}^{+} \mathrm{T}$ cells on immunohistological slices of myocardial autopsy samples from patients who died at various time points after MI were quantified as previously described (68). Furthermore, 9 patients for whom there was no pathological evidence of death from a cause related to MI were included 
as controls. Furthermore, we reexamined existing PET/CT data sets from patients who had recently had a MI and from patients without heart disease using a novel CXCR4-specific radioligand and depicted its expression in both med-LNs and the myocardium (69). Five patients who underwent CXCR4-directed PET/CT because of a suspected benign Conn's adenoma were included as controls. Detailed patient information is provided in Supplemental Tables 4 and 5.

\section{Data and material availability}

The transcriptomic data discussed in this study have been deposited in NCBI's Gene Expression Omnibus (GEO) database (GEO GSE116569; https://www.ncbi.nlm.nih.gov/geo/query/acc.cgi?acc=GSE116569). Likewise, the TCR-Seq data discussed in this study are accessible through the NCBI's GEO database (GEO GSE117076; https://www. ncbi.nlm.nih). The TCR-M mice used in this study were made available under a material transfer agreement with Kantonsspital St. Gallen (St. Gallen, Switzerland).

\section{Statistics}

Graphs of the data obtained from all the EMI studies were prepared to display the group mean values (bars), the SEM, and the distributions of each individual value. Statistical analyses were performed using GraphPad Prism software (version 7.0d, GraphPad Software), and the TCR-Seq data were statistically analyzed using MATLAB 2017a (MathWorks). A $P$ value of less than 0.05 was considered statistically significant. The statistical analyses of the data obtained from the clinical studies were performed using Statistical Packages for Social Sciences software (IBM SPSS 22.0.0.0, IBM Corp.). Levene's test for normality was applied and showed that the data were not normally distributed. Differences between groups containing continuous data were tested using the Mann-Whitney $U$ test, unless otherwise indicated. Fisher's exact test and the Freeman-Halton extension of Fisher's exact test were used to test the associations between categorical data. An Unpaired, 2-tailed $t$ test was used to compare the mean of 2 groups with data following a normal distribution. The results were considered significantly different if the 2 -sided $P$ value was less than 0.05 . The data are presented as box plots showing the medians, $25^{\text {th }}-75^{\text {th }}$ percentiles (boxes), and $5^{\text {th }}-95^{\text {th }}$ percentiles (whiskers).

\section{Study approval}

All animal procedures were approved by the local authorities (Landesverwaltungsamt Sachsen-Anhalt, Halle, Germany, and Regierung von Unterfranken, Würzburg, Germany) and were performed according to the guidelines of the Federation for Laboratory Animal Science Associations (FELASA) $(70,71)$. The study involving human autopsy samples was approved by and performed according to the guidelines of the ethics committee of Amsterdam UMC, location VUmc, Amsterdam Medical Center, and conformed to Declaration of Helsinki principles. [68Ga]Pentixafor was administered clinically on a compassionate use basis in compliance with section 37 of the Declaration of Helsinki and the German Medicinal Products Act (AMG section $132 \mathrm{~b}$ ) and in accordance with the responsible regulatory body (Regierung von Oberfranken, Bayreuth, Germany). All patients provided written informed consent prior to undergoing imaging procedures. Given the retrospective nature of this study, the local IRB of the University Hospital Würzburg waived the requirement for additional approval.

\section{Author contributions}

CG performed the epitope-mapping experiments. TCR-M cells were generated by BL and CGC. MR, MD, and GCR performed the cell transfers, mouse surgeries, and echocardiography. MD, MR, CK, and GCR performed the experiments and analyzed the data (immunization, flow cytometry, FACS, and histology). PS and GCR performed the transcriptomic analyses and analyzed the transcriptome readouts. GCR, MR, SF, and UH designed the murine studies. KGH and LB analyzed the LSFM samples. HWMN, ENTH, PAJK, AMVDL, and JJP acquired and analyzed the human MI autopsy samples. CL and MK acquired and analyzed the PET/CT data, and WRB and TR acquired and analyzed the CMR imaging data. NF and DR analyzed the TCR data, and CGC and BL generated the TCR-M mouse strain and contributed to the interpretation of the data. HJW developed and provided the CXCR4 PET tracer used in this study. All the coauthors contributed to the preparation of the manuscript.

\section{Acknowledgments}

We greatly appreciate the excellent technical assistance of Susanne Koch, Claudia Pilowski, Helga Wagner, Andrea Leupold, Elena Vogel, Jürgen Pinnecker, Mike Friedrich, Alexander Navarrete-Santos, and Vesselin Christov and the theoretical contributions provided by Gottfried Alber (Leipzig University) and Nelson Vaz (Federal University of Minas Gerais). The graphical abstract figure was prepared with the help of Servier Medical Art. GCR received funding from the Interdisciplinary Center for Clinical Research-Würzburg (IZKF-Würzburg, E-354), the Marija-Orlovic Foundation (Innovative Cardiology 2016 Prize), and the European Research Area Network - Cardiovascular Diseases (ERANET-CVD JCT2018, AIR-MI Consortium, grant 01KL1902), and from the German Research Foundation (DFG grant 411619907). UH received funding from the Deutsche Forschungsgemeinschaft (SFB688, TPA10), and KGH received funding from the Rudolf-Virchow Center. CG received a scholarship from the Deutsche Herzstiftung, and LB received a scholarship from the Deutsche Gesellschaft für Kardiologie. NF is supported by the David and Fela Shapell Family Institute for Preclinical Studies and the Florence Blau Charitable Trust and is the incumbent of the Eugene and Marcia Applebaum Professorial Chair.

Address correspondence to: Gustavo Ramos, Immunocardiology Lab, Am Schwarzenberg 15, Haus A15, D-97078 Würzburg, Germany. Phone: 49.931.201.46477; Email: ramos_g@ukw.de.
1. Amit U, et al. New role for interleukin-13 receptor $\alpha 1$ in myocardial homeostasis and heart failure. JAm Heart Assoc. 2017;6(5):e005108.

2. Monnerat G, et al. Macrophage-dependent IL-1 $\beta$ production induces cardiac arrhythmias in diabetic mice. Nat Commun. 2016;7:13344.
3. Hulsmans M, et al. Macrophages facilitate electrical conduction in the heart. Cell. 2017;169(3):510-522.e20.

4. Ramos GC, et al. Myocardial aging as a T-cell-mediated phenomenon. Proc Natl Acad Sci US A. 2017;114(12):E2420-E2429.
5. Hofmann U, Frantz S. Role of lymphocytes in myocardial injury, healing, and remodeling after myocardial infarction. Circ Res. 2015;116(2):354-367.

6. Hofmann U, Frantz S. Role of T-cells in myocardial infarction. Eur Heart J. 2016;37(11):873-879.

7. Ramos G, Hofmann U, Frantz S. Myocardial 
fibrosis seen through the lenses of T-cell biology. JMol Cell Cardiol. 2016;92:41-45.

8. Nunes-Silva V, Frantz S, Ramos GC. Lymphocytes at the heart of wound healing. Adv Exp Med Biol. 2017;1003:225-250.

9. Ibanez B, et al. 2017 ESC Guidelines for the management of acute myocardial infarction in patients presenting with ST-segment elevation: The Task Force for the management of acute myocardial infarction in patients presenting with ST-segment elevation of the European Society of Cardiology (ESC). Eur Heart J. 2018;39(2):119-177.

10. Ponikowski P, et al. 2016 ESC Guidelines for the diagnosis and treatment of acute and chronic heart failure: The Task Force for the diagnosis and treatment of acute and chronic heart failure of the European Society of Cardiology (ESC). Developed with the special contribution of the Heart Failure Association (HFA) of the ESC. Eur J Heart Fail. 2016;18(8):891-975.

11. Schwartz M, Moalem G, Leibowitz-Amit R, Cohen IR. Innate and adaptive immune responses can be beneficial for CNS repair. Trends Neurosci. 1999;22(7):295-299.

12. Harty M, Neff AW, King MW, Mescher AL. Regeneration or scarring: an immunologic perspective. Dev Dyn. 2003;226(2):268-279.

13. Burzyn D, et al. A special population of regulatory $\mathrm{T}$ cells potentiates muscle repair. Cell. 2013;155(6):1282-1295.

14. Hui SP, et al. Zebrafish regulatory T cells mediate organ-specific regenerative programs. Dev Cell. 2017;43(6):659-672.e5.

15. Maisel A, Cesario D, Baird S, Rehman J, Haghighi P, Carter S. Experimental autoimmune myocarditis produced by adoptive transfer of splenocytes after myocardial infarction. Circ Res. 1998;82(4):458-463.

16. Hofmann $\mathrm{U}$, et al. Activation of $\mathrm{CD} 4^{+} \mathrm{T}$ lymphocytes improves wound healing and survival after experimental myocardial infarction in mice. Circulation. 2012;125(13):1652-1663.

17. Ramos GC, et al. The autoimmune nature of post-infarct myocardial healing: oral tolerance to cardiac antigens as a novel strategy to improve cardiac healing. Autoimmunity. 2012;45(3):233-244.

18. Weirather J, et al. Foxp $3^{+} \mathrm{CD}^{+} \mathrm{T}$ cells improve healing after myocardial infarction by modulating monocyte/macrophage differentiation. Circ Res. 2014;115(1):55-67.

19. Bansal SS, et al. Activated T lymphocytes are essential drivers of pathological remodeling in ischemic heart failure. Circ Heart Fail. 2017;10(3):e003688.

20. Saxena A, et al. Regulatory $\mathrm{T}$ cells are recruited in the infarcted mouse myocardium and may modulate fibroblast phenotype and function. Am JPhysiol Heart Circ Physiol. 2014;307(8):H1233-H1242.

21. Van der Borght $\mathrm{K}$, et al. Myocardial infarction primes autoreactive $\mathrm{T}$ cells through activation of dendritic cells. Cell Rep. 2017;18(12):3005-3017.

22. Tang TT, et al. Regulatory T cells ameliorate cardiac remodeling after myocardial infarction. Basic Res Cardiol. 2012;107(1):232.

23. Skorska A, et al. The CD4(+) AT2R(+) T cell subpopulation improves post-infarction remodelling and restores cardiac function. J Cell Mol Med. 2015;19(8):1975-1985.
24. Borg N, et al. CD73 on T cells orchestrates cardiac wound healing after myocardial infarction by purinergic metabolic reprogramming. Circulation. 2017;136(3):297-313.

25. Laroumanie $\mathrm{F}$, et al. $\mathrm{CD}^{+} \mathrm{T}$ cells promote the transition from hypertrophy to heart failure during chronic pressure overload. Circulation. 2014;129(21):2111-2124.

26. Nevers T, et al. Left ventricular T-cell recruitment contributes to the pathogenesis of heart failure. Circ Heart Fail. 2015;8(4):776-787.

27. Kallikourdis M, et al. T cell costimulation blockade blunts pressure overload-induced heart failure. Nat Commun. 2017;8:14680.

28. Boag SE, et al. T lymphocytes and fractalkine contribute to myocardial ischemia/reperfusion injury in patients. JClin Invest. 2015;125(8):3063-3076.

29. Uhlén M, et al. Proteomics. Tissue-based map of the human proteome. Science. 2015;347(6220):1260419.

30. Wang P, Sidney J, Dow C, Mothé B, Sette A, Peters B. A systematic assessment of MHC class II peptide binding predictions and evaluation of a consensus approach. PLoS Comput Biol. 2008;4(4):e1000048.

31. Pummerer CL, et al. Identification of cardiac myosin peptides capable of inducing autoimmune myocarditis in $\mathrm{BALB} / \mathrm{c}$ mice. JClin Invest. 1996;97(9):2057-2062.

32. Krebs $\mathrm{P}$, et al. Molecular mapping of autoimmune $\mathrm{B}$ cell responses in experimental myocarditis. JAutoimmun. 2007;28(4):224-233.

33. Nindl V, et al. Cooperation of Th1 and Th17 cells determines transition from autoimmune myocarditis to dilated cardiomyopathy. Eur J Immunol. 2012;42(9):2311-2321.

34. Smith SC, Allen PM. Expression of myosin-class II major histocompatibility complexes in the normal myocardium occurs before induction of autoimmune myocarditis. Proc Natl Acad Sci U S A. 1992;89(19):9131-9135.

35. Murphy KM, Heimberger AB, Loh DY. Induction by antigen of intrathymic apoptosis of CD4+CD8+TCRlo thymocytes in vivo. Science. 1990;250(4988):1720-1723.

36. Hulsmans M, et al. Cardiac macrophages promote diastolic dysfunction. J Exp Med. 2018;215(2):423-440.

37. Yang X, et al. TCRklass: a new K-string-based algorithm for human and mouse TCR repertoire characterization. JImmunol. 2015;194(1):446-454.

38. Madi A, et al. T-cell receptor repertoires share a restricted set of public and abundant CDR3 sequences that are associated with self-related immunity. Genome Res. 2014;24(10):1603-1612.

39. Madi A, et al. $\mathrm{T}$ cell receptor repertoires of mice and humans are clustered in similarity networks around conserved public CDR3 sequences. Elife. 2017;6:e22057.

40. Holder A, Mirczuk SM, Fowkes RC, Palmer DB, Aspinall R, Catchpole B. Perturbation of the T cell receptor repertoire occurs with increasing age in dogs. Dev Comp Immunol. 2018;79:150-157.

41. Contento RL, et al. CXCR4-CCR5: a couple modulating T cell functions. Proc Natl Acad Sci U S A. 2008;105(29):10101-10106.

42. Chong SZ, Evrard M, Goh CC, Ng LG. Illuminating the covert mission of mononuclear phagocytes in their regional niches. Curr Opin Immunol. 2018;50:94-101.

43. Caron G, Le Gallou S, Lamy T, Tarte K, Fest T. CXCR4 expression functionally discriminates centroblasts versus centrocytes within human germinal center B cells. JImmunol. 2009;182(12):7595-7602.

44. Abbott JD, Huang Y, Liu D, Hickey R, Krause DS, Giordano FJ. Stromal cell-derived factor-1alpha plays a critical role in stem cell recruitment to the heart after myocardial infarction but is not sufficient to induce homing in the absence of injury. Circulation. 2004;110(21):3300-3305.

45. Heinze B, et al. Targeting CXCR4 (CXC chemokine receptor type 4) for molecular imaging of aldosterone-producing adenoma. Hypertension. 2018;71(2):317-325.

46. Ertl G, Frantz S. Wound model of myocardial infarction. Am J Physiol Heart Circ Physiol. 2005;288(3):H981-H983.

47. Yan X, et al. Temporal dynamics of cardiac immune cell accumulation following acute myocardial infarction. JMol Cell Cardiol. 2013;62:24-35.

48. Epelman S, Liu PP, Mann DL. Role of innate and adaptive immune mechanisms in cardiac injury and repair. Nat Rev Immunol. 2015;15(2):117-129.

49. Latet SC, Hoymans VY, Van Herck PL, Vrints CJ. The cellular immune system in the postmyocardial infarction repair process. Int $J$ Cardiol. 2015;179:240-247.

50. Prabhu SD, Frangogiannis NG. The biological basis for cardiac repair after myocardial infarction: from inflammation to fibrosis. Circ Res. 2016;119(1):91-112.

51. Van der Borght K, Lambrecht BN. Heart macrophages and dendritic cells in sickness and in health: A tale of a complicated marriage. Cell Immunol. 2018;330:105-113.

52. Nahrendorf M, Pittet MJ, Swirski FK. Monocytes: protagonists of infarct inflammation and repair after myocardial infarction. Circulation. 2010;121(22):2437-2445.

53. Frantz S, et al. Monocytes/macrophages prevent healing defects and left ventricular thrombus formation after myocardial infarction. FASEB J. 2013;27(3):871-881.

54. Mathes D, et al. CD4 ${ }^{+}$Foxp $^{+}$T-cells contribute to myocardial ischemia-reperfusion injury. J Mol Cell Cardiol. 2016;101:99-105.

55 . Wang YP, et al. Regulatory T lymphocytes in myocardial infarction: a promising new therapeutic target. Int J Cardiol. 2016;203:923-928.

56. Xiu J, et al. Prognostic value of myocardial perfusion analysis in patients with coronary artery disease: A Meta-Analysis. J Am Soc Echocardiogr. 2017;30(3):270-281.

57. Kallikourdis M. T cell responses to tumor: how dominant assumptions on immune activity led to a neglect of pathological functions, and how evolutionary considerations can help identify testable hypotheses for improving immunotherapy. Cancer Immunol Immunother. 2018;67(6):989-998.

58. Hu M, et al. Long-term cardiac allograft survival across an MHC mismatch after "pruning" of alloreactive $\mathrm{CD} 4 \mathrm{~T}$ cells. J Immunol. 2008;180(10):6593-6603.

59. Klingenberg R, et al. Clonal restriction and predominance of regulatory $\mathrm{T}$ cells in coronary thrombi of patients with acute coronary syn- 
dromes. Eur Heart J. 2015;36(17):1041-1048.

60. Heidecker B, et al. Transcriptomic biomarkers for the accurate diagnosis of myocarditis. Circulation. 2011;123(11):1174-1184.

61. Blohm JH, et al. Detection of clonal T-cell-receptor (TCR) Vbeta rearrangements in explanted dilated cardiomyopathy hearts by semi-nested PCR, GeneScan, and direct sequencing. Med Sci Monit Basic Res. 2013;19:111-117.

62. Lindskog C, et al. The human cardiac and skeletal muscle proteomes defined by transcriptomics and antibody-based profiling. BMC Genomics. 2015;16:475.

63. Lv H, Lipes MA. Role of impaired central tolerance to $\alpha$-myosin in inflammatory heart disease. Trends Cardiovasc Med. 2012;22(5):113-117.
64. Murphy KM. Janeway's Immunobiology. Oxford, United Kingdom and New York, New York, USA: Taylor \& Francis Group; 2011.

65. Wang Y, et al. C-X-C motif chemokine receptor 4 blockade promotes tissue repair after myocardial infarction by enhancing regulatory $\mathrm{T}$ cell mobilization and immune-regulatory function. Circulation. 2019;139(15):1798-1812.

66. Battaglia A, et al. Lymphocyte populations in human lymph nodes. Alterations in $\mathrm{CD}^{+}$ $\mathrm{CD}_{25^{+}} \mathrm{T}$ regulatory cell phenotype and T-cell receptor Vbeta repertoire. Immunology. 2003;110(3):304-312.

67. Ertl G, Frantz S. Healing after myocardial infarction. Cardiovasc Res. 2005;66(1):22-32.

68. van der Laan AM, et al. Monocyte subset accumulation in the human heart following acute myocardial infarction and the role of the spleen as monocyte reservoir. Eur Heart J. 2014;35(6):376-385.

69. Reiter T, et al. Imaging of C-X-C motif chemokine receptor CXCR4 expression after myocardial infarction with $\left[{ }^{68} \mathrm{Ga}\right]$ pentixafor-PET/CT in correlation with cardiac MRI. JACC Cardiovasc Imaging. 2018;11(10):1541-1543.

70. Voipio H-M, Baneux P, Gomez de Segura IA, Hau J, Wolfensohn S. Guidelines for the veterinary care of laboratory animals: report of the FELASA/ ECLAM/ESLAV Joint Working Group on Veterinary Care. Lab Anim. 2008;42(1):1-11.

71. Guillen J. FELASA guidelines and recommendations. JAm Assoc Lab Anim Sci. 2012;51(3):311-321. 